\title{
Transparency and distressed sales under asymmetric information
}

\author{
William Fuchs \\ Haas School of Business, University of California, Berkeley \\ ANIKO ÖRY \\ School of Management, Yale University \\ ANDRZEJ SKRZYPACZ \\ Graduate School of Business, Stanford University
}

\begin{abstract}
We analyze price transparency in a dynamic market with private information and interdependent values. Uninformed buyers compete inter- and intra-temporarily for a good sold by an informed seller suffering a liquidity shock. We contrast public versus private price offers. With two opportunities to trade, all equilibria with private offers have more trade than any equilibrium with public offers; under some additional conditions, we show Pareto dominance of the private-offers equilibria. If a failure to trade by the deadline results in an efficiency loss, public offers can induce a market breakdown before the deadline, while trade never stops with private offers.
\end{abstract}

KEYWORDs. Adverse selection, transparency, distress, market design, volume.

JEL CLASSification. D82, G14, G18.

\section{IntRoduction}

When designing an exchange venue or regulating markets, an important consideration is to what extent sellers' willingness to sell or buyers' willingness to buy are reflected in information available to other market participants. One natural and important source of available information is the set of previous offers that were not accepted. For example, venues referred to as "dark pools" hide information about the order book, while

William Fuchs: wfuchs@haas . berkeley .edu

Aniko Öry: aniko. oery@yale .edu

Andrzej Skrzypacz: skrz@stanford .edu

We thank Emmanuel Fahri, Brett Green, Terry Hendershot, Benjamin Hermalin, Johannes Hörner, Giorgio Martini, Christine Parlour, Alessandro Pavan, Nicolas Vieille, Pavel Zryumov, and participants of the 8th Annual Paul Woolley Center Conference at LSE, Central European University, CERGE, 2013 EEA Meetings, Federal Reserve Bank of Philadelphia, Jackson Hole Finance Conference, 23rd Jerusalem Summer School in Economic Theory, NBER Corporate Finance Meetings, University of Munich, UC Berkeley Theory Lunch, and Yale. We are also grateful for support for this project from the NSF.

Copyright $\odot 2016$ William Fuchs, Aniko Öry, and Andrzej Skrzypacz. Licensed under the Creative Commons Attribution-NonCommercial License 3.0. Available at http: //econtheory . org.

DOI: $10.3982 / \mathrm{TE} 2237$ 
order books are visible in the New York Stock Exchange (NYSE). ${ }^{1}$ Similarly, eBay now allows buyers and sellers to negotiate on the side without displaying those negotiations to other prospective bidders. Importantly, the lack of transparency about unconsummated offers changes the ability of a seller to endogenously signal her type by not accepting a given offer. We analyze the consequences of this on trading dynamics and welfare within a dynamic market for lemons with deadlines.

We consider a problem of an owner of an indivisible durable asset who suffers a liquidity shock. Due to the liquidity shock, the seller's present value of the good drops to a lower level than the true value of the good. Hence, she would like to sell the asset to a buyer who is not facing a liquidity shock. The problem is that, usually, the owner of the asset is better informed about its quality. Thus, although there are gains from trade, buyers face an adverse selection problem. As first stressed by Akerlof (1970), if there is only one opportunity to trade, competitive buyers are only willing to pay the expected valuation of the asset conditional on the seller accepting the offered price. If the adverse selection problem is sufficiently strong, some types will choose not to sell in equilibrium. In Akerlof's model there is only one opportunity to sell the asset. In a dynamic setting, in which sellers get several chances to sell their goods, the alternative to not selling in the current period is to sell in the future. As a result, inefficiencies can arise from either failure to trade or delay in trade with some seller types.

When deciding whether to delay trade, the seller considers the current price offer and future expected prices. In turn, to set prices, buyers must form beliefs about the type of seller they are facing. The observability of past price offers clearly affects the available information buyers have based on which they update their beliefs. If the seller knows that future buyers will not observe today's price offers, then she cannot use rejections of high price offers to signal to those buyers that she is a high type. Thus, observability of price offers can change equilibrium dynamics and total welfare dramatically.

We analyze a two-period model with a long-lived, privately informed seller and a competitive market of buyers in every period (modeled as a number of short-lived buyers competing in prices in every period). We consider two opposite information structures: transparent (public offers), in which all buyers observe past price offers and opaque (private offers), in which buyers do not observe past rejected offers.

Moreover, by allowing for a fraction of surplus to be lost at the deadline, we capture an additional notion of the seller's distress. For example, financial distress can lead to forced liquidation at some deadline if liquidity cannot be restored by selling assets. In such a case, when the deadline is reached, the opportunity to trade disappears. Similarly, a profitable investment opportunity that the seller wants to finance with the proceeds from the sale of the asset can diminish at a deadline, in which case distress is caused by a temporary opportunity rather than the fear of default. This can create a deadline effect resulting in the seller trading with a high probability just at the deadline. On the flip side, the deadline could represent the time at which a report about the quality of the asset becomes public, such as the granting or denial of Food and Drug

\footnotetext{
${ }^{1}$ Bloomberg reports that off-exchange platforms including dark pools handled a daily average of 34 percent of U.S. stock trading in 2015.
} 
Administration (FDA) approval, restoring symmetric information and allowing efficient trading at that point. This reduces the eagerness to trade just before the deadline. By introducing a parameter for the degree of distress at the deadline, we illustrate a novel difference between transparent and opaque markets: With public offers (see Proposition 1), distress at the deadline endogenously leads to a trading impasse (illiquidity) before the deadline. In contrast, with private offers (see Proposition 2), there cannot be a trading impasse (i.e., there is trade with positive probability in every period). Thus, price transparency is a particularly important issue if distress at the deadline is present.

We also analyze the welfare consequences of price transparency (see Theorem 1). First, we show that in an opaque market, prices in the second period are higher, resulting in more total trade. This implies that all seller types that would have traded in the second period in a transparent market must be better off. If, in addition, there are also weakly higher prices in the first period, then the opaque market Pareto-dominates the transparent market. This is the case when not trading by a deadline imposes an efficiency loss and discounting between periods is low (trading is frequent). We also obtain a complete characterization of equilibria when gains from trade are linear in valuation and the distribution of valuations is uniform (see Theorem 3). In that case, private offers strictly Pareto-dominate public offers if discounting between trade opportunities is sufficiently low, even if missing the deadline would not additionally reduce welfare. If discounting between the two periods is large, the two information structures yield the same equilibrium outcome. Only if discounting between opportunities to trade is small will equilibrium prices with private offers differ from those with public offers, with buyers randomizing between several price offers so that market prices appear volatile.

What makes the markets operate differently in these two information regimes? In a transparent market, buyers can observe all previous price offers and thereby learn about the quality of the good through two channels: the number of rejected offers (time on the market) and the price levels that have been rejected by the seller. By rejecting a high offer, the seller can send a strong signal to future buyers that she is of a high type. For example, in transparent exchanges, sellers try to influence prices by taking advantage of the observability of order books. In contrast, in an opaque market, in which buyers cannot observe previously rejected prices, the seller signals only via delay. Intuitively, private offers generate more trade because the seller's continuation value is independent of the current price offer, while with public offers it increases in prices. Thus, sellers are more reticent to accept public offers than to accept private offers (leading to less trade in the transparent market). ${ }^{2}$

For the difference in equilibrium dynamics, the frequency of trade (or, equivalently, the discounting between periods) plays an important role. Any effect on future offers

\footnotetext{
${ }^{2}$ In light of the existing literature, this paper reveals that the effect of transparency on price dynamics depends on the microstructure of the market. For example, in our model, the observation that any purestrategy equilibrium prices in a game with private offers are also supportable as equilibrium prices in a game with public offers is true because we have assumed intra-period competition. In Kaya and Liu (2015) there is one buyer per period and hence competition is only inter-period. In that case the games with private and public offers have different pure-strategy equilibria. The reason for the difference is that a monopolistic buyer would have a profitable deviation to a lower price if prices became transparent. In our model intra-period competition implies that a lower-than-equilibrium price is rejected for sure.
} 
will be more relevant the closer is the next opportunity to trade. We show that transparency only affects the amount of inefficient delay whenever the time between price offers is small (see Theorem 2). As we show, the intuition of Theorem 2 extends to a general multiperiod setting: pure-strategy perfect Bayesian equilibria (PBE) with public offers always exist and they coincide with PBE with private offers if the discounting between two periods is large (see Theorem 4). However, there cannot be pure-strategy PBEs in the game with private offers if the discounting between two periods is small, because in a pure-strategy equilibrium, sellers are willing to accept higher prices with private offers (not being able to signal to tomorrow's buyers), which makes it profitable for today's buyers to deviate to higher prices. Consequently, the two information structures result in different trading patterns only in high-frequency markets. Given this result and the intuition for our welfare results, we believe that the welfare results also extend to a multiperiod model. We are able to show that the Pareto ranking holds strictly for sufficiently high discount factors and some distress at the deadline (see Remark 2). However, due to multiplicity of equilibria (in particular with private offers) we have not been able to formally establish that the ranking is never reversed for infrequent trading.

\section{Related literature}

The closest paper to ours in the economics literature is Hörner and Vieille (2009) (HV hereinafter). They are also interested in comparing trading dynamics in a dynamic lemons market with public versus private offers. Our model differs from theirs in that we allow for a deadline effect and we consider multiple buyers in every period. The lack of intra-period competition in HV introduces Diamond paradox effects (Diamond 1971), making it much more difficult to isolate the effect of transparency and resulting in a "paradoxical" equilibrium with public offers: the first offer is rejected with positive probability and all other offers are rejected with probability 1 . Instead, in our model, in the equilibrium with public offers, trade occurs gradually over time. By eliminating the Diamond paradox effects and adding a deadline, we can derive additional welfare results. Although HV show that private offers lead to more trade, in general it is not obvious whether their model would lead to an efficiency ranking. It is possible that the endogenous trading impasse that arises with public offers in their model is actually valuable since it serves as a commitment device where sellers know that they either trade in the first period or never again. Indeed, as shown in Fuchs and Skrzypacz (2015), efficiency is actually enhanced when the privately informed seller is exogenously restricted to only one opportunity to sell. Last, due to the infinite horizon model, in HV's work there are no counterparts to our results regarding distress at the deadline.

Another interesting prior comparison between private and public offers goes back to Swinkels (1999). He looks at a dynamic version of the Spence signaling model where potential employers are allowed to make private offers to the "students" at any time. Swinkels shows that in this case the unique equilibrium outcome is a pooling equilibrium with all students being hired at time 0 . This, he points out, is in direct contrast to Nöldeke and Van Damme (1990), who show that, with public offers, the unique equilibrium to survive the NWBR refinement is a separating equilibrium where the high types 
go to school just long enough to credibly separate themselves from the low types. The main difference between both these papers and ours is similar to the difference between Spence and Akerlof. In our model, the adverse selection problem is stronger and hence, the buyers would not be willing to buy at the price necessary to get all sellers to sell, even if offers were private. ${ }^{3}$

Our result about the nonexistence of pure-strategy equilibria in the private-offers case is related to the result in Kremer and Skrzypacz (2007), who study a dynamic version of the education signaling model with private offers, a finite horizon, and the type being (partially or fully) revealed in the last period (which endogenously creates adverse selection). They show that there do not exist fully separating equilibria in a game with a continuum of types and continuous time. The intuition in their proof for why separation is not possible is similar to our intuition for why pure-strategy equilibria do not exist. In particular, with private offers, sellers follow a reservation price strategy and the reservation prices are equal to the continuation payoffs, which are independent of current prices. Kremer and Skrzypacz (2007) show that if the equilibrium was separating, in continuous time the reservation prices would have zero derivative at the lowest type, resulting in a perfectly elastic supply. That in turn would lead to a profitable deviation for the buyers (who with a very small price increase could attract strictly better types). In our discrete-time model we show that if the candidate equilibrium of the game with private offers is in pure strategies, and the discount factor is high enough (or periods are short enough), then equilibrium supply is sufficiently elastic to create similar profitable deviation for the buyers.

More recently, Kim (2015) compares three different information structures in a continuous time setting in which many sellers and buyers, who arrive over time at a constant rate, match randomly. In every match, the buyer makes a price offer that the seller can accept or reject. The type space of the seller is binary. Instead of looking at observability of past offers, he compares steady state equilibria in settings in which buyers do not observe any past histories to settings in which the time on the market or the number of past matches can be observed by buyers. The welfare ordering is not as clear cut as in our paper. He shows that with small frictions, the setup in which only the time on the market is observable is optimal, while with large frictions, the welfare ordering can be reversed.

For repeated first-price auctions, Bergemann and Hörner (2014) consider three different disclosure regimes and show that if bidders learn privately whether they won, welfare is maximized and information is eventually revealed. Kaya and Liu (2015) compare public versus private offers in a setup with independent values with a long-lived seller and one buyer per period. Asriyan et al. (2015), in contrast, are concerned with the transparency of transactions and the effects of the ensuing information spillovers if there are several assets being sold and their values are correlated.

Besides our contribution regarding the implications of transparency, our paper also contributes to the literature on dynamic lemons markets in general. Deneckere and Liang (2006) consider an infinite horizon bargaining situation, i.e., one long-lived buyer

\footnotetext{
${ }^{3}$ This is also what causes delays in trade in the bargaining model by Deneckere and Liang (2006).
} 
and one long-lived seller, with correlated valuations. They show that even in the limit as the discount factor goes to 1 , there can be an inefficient delay of trade unlike that predicted by the Coase conjecture. ${ }^{4}$ Janssen and Roy (2002) obtain similar results with a dynamic competitive lemons market with discrete time, infinite horizon, and a continuum of buyers and sellers. While in their model both market sides compete, we assume that there is only one seller. Unlike most previous papers that consider slightly different market structures, we are able to provide a more complete characterization of equilibria in mixed strategies with private offers. This makes it possible to understand these kinds of equilibria in more detail. For example, we show that "non-offers," i.e., offers that are never accepted, are always part of an equilibrium in the first period if offers are private and offers are frequent.

A number of recent papers work directly in continuous time and, rather than modeling buyers as strategic, they assume there is some competitive equilibrium price path. This paper is a complement to those papers. For example, one can understand the No Deals Condition in Daley and Green (2012) as arising from private offers and the Market Clearing Condition in Fuchs and Skrzypacz (2015) as arising in a setting with public offers. $^{5}$

The finance literature has also looked at transparency questions. In particular, our model is more directly related to what is referred to as pre-trade transparency. Most of the theoretical and empirical work has focused on order book transparency. ${ }^{6}$ The two main trade-offs regarding transparency within this literature are the "advertising" and the "information" effects. The former refers to the notion that when the desire to trade is made public, then it is beneficial because more potential counterparties become aware and might participate. The latter effect refers to the information revealed about the underlying asset that the poster of the offer has. Importantly, it leads to less trade with a public order book since traders do not want to reveal private information to the market. Neither of these effects is present in our model since the size of the market is fixed and all the information is in the hands of the seller, who does not make any offers. This allows us to highlight the novelty of the dynamic signaling effect we uncover in our paper.

\section{Model AND PRELIMINARies}

\subsection{General setup}

A seller has an asset that she values at $c$, which is her private information and which is distributed according to a cumulative distribution function $F$ on [0,1]. One can think of the asset as giving an expected cash flow each period and as $c$ being its present value for the seller. ${ }^{7}$ There are two opportunities to trade with two short-lived buyers arriving

\footnotetext{
${ }^{4}$ See also Fuchs and Skrzypacz (2013b).

${ }^{5}$ We have benefited from discussions with Brett Green on these issues.

${ }^{6}$ See, for example, Buti and Rindi (2013), Flood et al. (1999), Boehmer et al. (2005), Madhavan et al. (2005), and Pancs (2014).

${ }^{7}$ Alternatively, and mathematically equivalently, the model can be rewritten so that $c$ can be thought of as the cost of producing the asset.
} 
in each period $t \in\{1,2\} .^{8}$ The buyers make simultaneous price offers to purchase the asset. ${ }^{9}$ The value of the asset for the buyers is given by $v(c)$ with $v^{\prime}(c)>0, v(1)=1$, and gains from trade $v(c)-c$ are strictly positive for all $c \in[0,1) .{ }^{10}$ The gains from trade can arise from the fact that the seller has received some liquidity shock and thus values the asset below its fundamental value. The game ends as soon as the good is sold. If trade has not taken place by the end of the second period, then the seller obtains a fraction of the gains from trade: $(1-\alpha)(v(c)-c)$ with $\alpha \in[0,1]$. One can think of $\alpha$ as a measure of distress at the deadline. If $\alpha=0$, there is no efficiency loss once the deadline is reached, i.e., the only source of inefficiency is delay in trade. For example, this could capture the possibility of the private information being short-lived, the deadline representing the time at which the private information becomes public. This could be the result of some government stress test or the uncertainty of regulatory approval (e.g., by the FDA) being resolved. If $\alpha>0$ there is additional efficiency loss if trade does not take place before the deadline. This can arise in a situation in which financial distress can lead to forced liquidation at the deadline. If the deadline is reached, the value of assets is evaluated and the assets are sold while only a fraction of the proceeds from sale are credited to the seller. When there is no opportunity to trade after period 2, we have $\alpha=1$. This captures, for example, a situation in which the seller has an investment opportunity and is required to raise the necessary funds by a certain date.

The seller discounts payoffs according to a discount factor $\delta \in(0,1)$. All players are risk neutral. Given the seller's type is $c$ and agreement over a price $p$ is reached in period $t$, the seller's (period 1 present value) payoff is $\left(1-\delta^{t-1}\right) c+\delta^{t-1} p$; a buyer's payoff is $v(c)-p$ if he gets the good and 0 otherwise. If there is no trade, the seller's payoff is $c+\delta^{2}(1-\alpha)(v(c)-c)$. Without loss of generality, we restrict prices to be in $[0, v(1)]$ since it is a dominant strategy for the seller to reject any negative price, and for any buyer it is a dominated strategy to offer any price higher than $v(1)$.

We explore two different information structures. In the public-offers case, period 2 buyers observe rejected offers from period 1. In contrast, with private offers, period 2 buyers are aware that the seller has rejected all offers in period 1 but do not know what those offers actually were.

Period 2 buyers' beliefs about the seller types they are facing is characterized by a cumulative distribution function (cdf) denoted by $F_{2}(c)$. Without loss of generality, we assume that the seller responses are independent of buyer identity. That is, conditional on receiving the same price offer, she treats both buyers equally.

Finally, we make a regularity assumption that

$$
\frac{f(c)}{F(c)}(v(c)-c)
$$

\footnotetext{
${ }^{8}$ In Section 4 we extended some results to more than two periods.

${ }^{9}$ The analysis is the same if there are more than two buyers since the buyers compete in a Bertrand fashion.

${ }^{10}$ In most of the paper we assume $v(1)=1$ to rule out the possibility of trade ending before the last period. This allows us to avoid making assumptions about out-of-equilibrium-path beliefs if the seller does not sell by $t$ even though in equilibrium all seller types are supposed to trade. If $v(1)>1$ but $\delta$ is large enough so that not all types trade in equilibrium, our analysis still applies.
} 
is strictly decreasing and that

$$
v^{\prime \prime}(c) \geq 0 .
$$

This implies that a one-shot game would have a unique equilibrium and that a zeroprofit condition must be satisfied in both periods for both information structures. ${ }^{11}$

\subsection{Equilibrium notion}

We are interested in characterizing perfect Bayesian equilibria (PBE) of the two games. A PBE of a given game is given by (possibly mixed) pricing strategies for the two buyers in each period, a sequence of acceptance rules of the seller, and the buyers' beliefs $F_{2}$ at the beginning of period 2 , satisfying the following three conditions:

C1. Any price offer in the support of a buyer's strategy must maximize the buyer's payoff conditional on the seller's acceptance rule, the other buyer's strategy, and the buyer's belief $F_{t}(c)$, where $F_{1}(c)=F(c)$ is the common prior.

C2. Buyers' beliefs $F_{2}$ are updated (whenever possible) according to Bayes' rule taking the seller's and the other buyers' strategies as given. In the public-offers game, beliefs are updated conditional on the offered prices in period 1.

C3. The seller's acceptance rule maximizes her profit taking into account the impact of her choices on the agents' updating and the future offers she can expect to receive as a result.

In the game with private offers, equilibrium strategies and beliefs depend only on the calendar time. In the game with public offers, period 2 strategies and beliefs depend also on the publicly observed prices offered in period 1 . With public offers, deviations from equilibrium price offers are observed by future buyers and induce different continuation play. With private offers, off-equilibrium price offers do not change the continuation play.

\subsection{Preliminaries}

As in other similar dynamic games, in equilibrium, the seller's acceptance rule can be characterized by a cutoff strategy. More precisely, given any history and highest price offer $p$, there exists a cutoff $k_{t}(p)$ such that sellers with valuation above cutoff $k_{t}(p)$ reject a price offer $p$ in period $t$ while sellers with valuation less than $k_{t}(p)$ accept it. In the bargaining literature, it is the better types that accept first and this property is known as the skimming property. Since here it is the worse types that trade first, we call it reverse skimming instead.

Lemma 1 (Reverse-skimming property). In any continuation equilibrium with either type of information structure, the following statement must hold: For any highest price offer $p$ in period $t$, there exists a cutoff type $k_{t}(p)$ such that a seller of type c accepts $p$ if $c<k_{t}(p)$ and rejects $p$ if $c>k_{t}(p){ }^{12}$

\footnotetext{
${ }^{11}$ If $\alpha=1$, assumption (2) can be dropped.

${ }^{12}$ Note that $k_{2}(p)$ is independent of the price history.
} 
See the Appendix for a formal proof. This lemma holds independently of the information structure in place (although the cutoffs may differ). The intuition for the lemma is straightforward. If a type- $c$ seller is willing to accept a price that, if rejected, would induce a given future price path, then all lower type sellers would also be willing to accept that price rather than wait for a price on that path because their flow payoff from possessing the asset is smaller.

A buyer's expected profit conditional on his offer being accepted is given by ${ }^{13}$

$$
\Pi_{t}\left(p ; F_{t}\right)=\int_{0}^{k_{t}(p)}(v(c)-p) d F_{t}(c) .
$$

Thanks to the reverse-skimming property, if past prices are observed publicly, the belief about the remaining seller types in period 2 is given by a single cutoff $k_{1}(p)$. Therefore, with public offers, if $p$ is the highest price offer observed in period 1 , then $F_{2}$ is just $F_{1}$ truncated to $\left[k_{1}(p), 1\right]$. In contrast, with private offers, if period 1 buyers play mixed strategies, period 2 buyers have nondegenerate beliefs over the possible cutoffs induced by period 1 prices. In that case, we denote the cdf representing the distribution of cutoffs after period 1 from period 2 buyers' point of view by $K_{1}:[0,1] \rightarrow[0,1]$. The probability density function (pdf) of the equilibrium belief, $f_{2}(c)$, is then given by Bayes' rule:

$$
f_{2}(c)=\int_{0}^{c} \frac{1}{1-F(\tilde{k})} d K_{1}(\tilde{k})
$$

LeMma 2 (Zero profit). In any equilibrium, buyers must make zero profits in both periods with both information structures.

We prove this lemma together with the following lemma, which shows that in equilibrium each cutoff can only be induced by a single price. Let $\kappa_{2}(k)$ be the period 2 cutoff of the continuation equilibrium given the period 1 cutoff is believed to be $k$ (which we show is unique).

Lemma 3 (Inverse supply). (i) Private offers. With private offers, on equilibrium path, there exists a unique price $p_{t}(k)$ that results in a given cutoff seller type $k$. The function $p_{t}(\cdot)=k_{t}^{-1}(\cdot)$ is increasing and continuous, and is given by

$$
p_{1}(k)=\delta \underbrace{\left[\left(\int_{k}^{1} p_{2}(\tilde{k}) d K_{2}(\tilde{k})\right)+K_{2}(k) p_{2}(k)\right]}_{\text {continuation payoff }}+\underbrace{(1-\delta) k}_{\text {utility from keeping the good }},
$$

where $K_{2}$ represents the cdf of the distribution of period 2 equilibrium cutoffs and

$$
p_{2}(k)=\delta(1-\alpha) v(k)+(1-(1-\alpha) \delta) k .
$$

\footnotetext{
${ }^{13}$ The expected profit of the buyer is the probability that he has the higher offer, or that he wins in case of a tie, times $\Pi_{t}\left(p ; F_{t}\right)$.
} 
(ii) Public offers. Consider an equilibrium with public offers. After any history, there is a unique price $p_{t}(k)$ at which the type- $k$ seller is the highest type accepting the price. The function $p_{t}(k)$ is increasing and is given by

$$
p_{1}(k)=\delta p_{2}\left(\kappa_{2}(k)\right)+(1-\delta) k
$$

and

$$
p_{2}(k)=\delta(1-\alpha) v(k)+(1-(1-\alpha) \delta) k
$$

The formal proof of the two lemmas is presented in the Appendix, but we provide some intuition here. From now on we call $p_{t}(\cdot)$ the inverse supply function defined in this lemma. It is derived from the seller's indifference condition in each period as described in this lemma.

In period 2, the unique price that results in cutoff type $k$ is the same for both information structures (since the seller continuation payoff is independent of the history). However, in period 1 , the seller's strategy and, hence, $p_{1}(k)$ are different across information structures.

With private offers, period 1 prices do not affect $F_{2}$ or the continuation play. As a result, the continuation payoff in (3) is independent of past cutoffs. The first part of the continuation payoff $\int_{k}^{1} p_{2}(\tilde{k}) d K_{2}(\tilde{k})$ is nothing but the expected price the seller can get if she sells the asset in period 2. The term $p_{2}(k)$ is the expected payoff that a type- $k$ seller can expect if she does not sell tomorrow either, which happens with probability $K_{2}(k)$. The term $(1-\delta) k$ represents the payoff of a type- $k$ seller if she held on to the good for exactly one more period. This total expected benefit from waiting must correspond to the payoff from selling today (at $p_{1}(k)$ ).

With public offers, however, period 1 prices can affect period 2 price offers, which makes the argument more evolved. We show that the period 2 cutoff of the continuation game, given that period 2 buyers believe the cutoff type after period 1 is $k$, increases in $k$. As a result, $k_{t}(p)$ is increasing and an inverse supply function exists.

As a consequence of Lemma 3, one can think of buyers essentially choosing cutoffs instead of prices given the seller's cutoff strategy $k_{t}(\cdot)$. More precisely, we can write a buyer's expected profit conditional on his offer being accepted, if he bids a price $p=$ $k_{t}^{-1}(k)$, and given that buyers believe that current cutoffs are distributed according to a $\operatorname{cdf} K$, as

$$
\pi_{t}(k ; K)=\int_{0}^{k} \int_{0}^{c} \frac{1}{1-F(\tilde{k})} d K(\tilde{k}) \cdot\left(v(c)-p_{t}(k)\right) \cdot f(c) d c .
$$

If $K$ has its entire mass on a singleton $\ell$ (which is always the case with public offers), then we write $\pi_{t}(k ; \ell)$ instead of $\pi_{t}(k ; K)$, abusing notation slightly. In particular, in period $1, \pi_{1}(k ; 0)=\int_{0}^{k}\left(v(c)-p_{1}(k)\right) \cdot f(c) d c$, where $p_{1}(k)$ varies across the two information structures. 


\section{Distress, transparency, AND Welfare}

In this section we present all our main results. We are interested in two types of questions. First, how do the two information structures compare in terms of welfare (Theorem 1) and second, how do equilibria differ with the two information structures (Theorems 2 and 3$)$.

\subsection{General results}

A full characterization of equilibria, in particular with private offers, is difficult because, as we show, buyers play mixed strategies and the equilibrium is generally not unique. ${ }^{14}$ Nevertheless, even without an explicit characterization of equilibria with private and public offers, we can show that all equilibria with private offers result in more trade than all equilibria with public offers. We also present sufficient conditions under which the private-offers regime Pareto-dominates the public-offers regime.

THeOREM 1. Consider an arbitrary equilibrium with public offers and an arbitrary equilibrium with private offers. Then the following statements hold:

(i) Expected second period prices are (weakly) higher and there is more total trade with private offers.

(ii) All types that, with public offers, either trade in the second period or wait until the deadline are in expectation (weakly) better off when offers are made privately.

(iii) If the first period expected prices are weakly higher in the equilibrium with private offers, then that equilibrium Pareto-dominates the equilibrium with public offers.

(a) For any given $\alpha>0$, there exists a $\delta^{* *}(\alpha)$ such that if $\delta>\delta^{* *}(\alpha)$, then the first period expected prices are weakly higher in the equilibrium with private offers and the private-offers equilibrium strictly Pareto-dominates the public-offers equilibrium.

(b) For $c \sim U[0,1]$ and linear $v$, there exists a $\delta^{*}$ such that the first period expected prices are weakly higher in the equilibrium with private offers and the privateoffers equilibrium strictly Pareto-dominates the equilibrium with public offers for any $\alpha \in[0,1]$ and $\delta \in\left(\delta^{*}, 1\right]$. For $\delta<\delta^{*}$, both equilibrium outcomes coincide.

Note that the statements in this theorem are true for any two equilibria with public and private offers, respectively, which allows us to provide comparisons for the whole sets of equilibria instead of relying on any selection.

We present the proof of (i) and (ii) here and show only the first part of (iii), i.e., the Pareto-ranking under the premise that the expected price in the first period with private

\footnotetext{
${ }^{14} \mathrm{An}$ explicit characterization of equilibria if valuations are linear and costs are uniformly distributed is presented in Section 3.3.
} 
offers is weakly higher than the expected price with public offers. We then show in Sections 3.2 and 3.3, that the premises in (iii) (a) or (b) imply weakly higher expected prices in period 1 with private offers.

Proof of Theorem 1. First, note that with public offers, we can restrict attention to pure-strategy equilibria because for any mixed-strategy equilibrium, one can construct a pure-strategy equilibrium that Pareto-dominates it. Such a pure-strategy equilibrium can be constructed as follows. The period 1 price $p_{1}^{*}$ is the largest price in the support of period 1 prices in the mixed-strategy equilibrium and must also result in the largest period 1 cutoff $k_{1}^{*}$. By the regularity assumption that (1) is decreasing and (2), there is only one price $p_{2}$ that satisfies the period 2 zero-profit condition, given any period 1 cutoff $k_{1}$. Then, given any period 1 cutoff $k>k_{1}$, profits are greater than zero at $p_{2}$. Hence, the period 2 price $p_{2}^{*}$ following the period 1 cutoff $k_{1}^{*}$ must be the largest period 2 price that is chosen with positive probability in the mixed equilibrium. As a result, the pure-strategy equilibrium given by price offers $p_{1}^{*}=p_{1}\left(k_{1}^{*}\right)$ and $p_{2}^{*}=p_{2}\left(k_{2}^{*}\right)$ exists and Pareto-dominates the mixed equilibrium.

Let us consider a public-offers equilibrium with cutoff types $k_{1}^{*}$ and $k_{2}^{*}$. If offered the equilibrium price $p_{1}^{*}$ in period 1 , the continuation payoff of type $c$ after rejecting the offer is given by

$$
V\left(c ; p_{1}^{*}\right) \equiv \delta \max \left\{p_{2}^{*}, p_{2}(c)\right\}+(1-\delta) c .
$$

Note that by definition $V\left(k_{1}^{*} ; p_{1}^{*}\right)=p_{1}^{*}$. In a private-offers equilibrium, the continuation payoff of type $c$ after rejecting an offer in period 1 is independent of the price in period 1 and is given by

$$
W(c) \equiv p_{1}(c)=\delta\left[\left(\int_{c}^{1} p_{2}(\tilde{k}) d K_{2}(\tilde{k})\right)+K_{2}(c) p_{2}(c)\right]+(1-\delta) c .
$$

Assume $W\left(k_{1}^{*}\right)<V\left(k_{1}^{*} ; p_{1}^{*}\right)=p_{1}^{*}$ can be shown to lead to a contradiction. ${ }^{15}$ We can thus focus on the case $W\left(k_{1}^{*}\right) \geq V\left(k_{1}^{*} ; p_{1}^{*}\right)$. In this case, for all $c \leq k_{2}^{*}$, since the equilibrium with private offers might involve mixing in the second period and might result in the seller deciding not to sell (if the second period realized offer is low), the derivative of the continuation value with respect to type is higher:

$$
\frac{\partial}{\partial c} W(c)=1-\delta+\delta K_{2}(c)\left(1-\delta+\alpha \delta+(1-\alpha) \delta v^{\prime}(c)\right) \geq 1-\delta=\frac{\partial}{\partial c} V\left(c ; p_{1}^{*}\right) .
$$

Hence, all seller types $k \in\left[k_{1}^{*}, k_{2}^{*}\right]$ have a better outside option with private offers when rejecting the period 1 price, which implies that all types $k \in\left[k_{1}^{*}, k_{2}^{*}\right]$ are better off with private offers. Sellers with $k \geq k_{2}^{*}$ wait until the deadline with public offers. They always have this option with private offers as well and can even be better off if they see a preferable price before. Thus, there must be more trade with private offers in period 2 .

\footnotetext{
${ }^{15} \mathrm{~A}$ lower continuation value with private offers would imply acceptance by types higher than $k_{1}^{*}$ in the first period, which in turn must imply higher prices in period 1 and, therefore, also in period 2 . This would imply $W\left(k_{1}^{*}\right)>V\left(k_{1}^{*} ; p_{1}^{*}\right)$.
} 
Sellers in $\left[k_{1}^{*}, 1\right]$ are better off since buyers break even and surplus for the seller is derived from trade, where more surplus can only be achieved with more trade. Given that the seller's reservation price in the second period is independent of the information structure, more trade can only be achieved with higher average prices, concluding the proof of (i) and (ii). If expected period 1 prices are higher with private offers than with public offers, then all seller types $k<k_{1}^{*}$ are also better off with private offers.

The proof and intuition for (iii) (a) is deferred to Section 3.2 where we discuss the role of distress $\alpha$. The proof and intuition for (iii) (b) is deferred to Section 3.3 because it requires a full characterization of mixed-strategy equilibria that is presented there.

Intuitively, we believe that this result is more general because, given that in expectation the pool of sellers in period 2 is higher with private offers, there should be more trade (and thus, higher prices) in period 1 with private offers. Nevertheless, this is hard to prove. The difficult step is to show that seller types $k<k_{1}^{*}$ are weakly better off. Except for the case of distress with high $\delta$ (see Section 3.2 below) in general, this requires an explicit characterization of the distribution of first period prices (for all possible equilibria) with private offers. Constructing all mixed-strategy equilibria with private offers is extremely challenging and we have not been able to do so beyond the linear case. In the linear case (see Section 3.3 below) payoffs become quadratic functions, which give us sufficient structure to pin down expected period 1 trade and to show that it is higher than $k_{1}^{*}$.

REMARK 1. A noteworthy consequence of Theorem 1 is that there can exist at most one pure-strategy equilibrium with private offers. This follows because any private-offers pure-strategy equilibrium corresponds to a public-offers pure-strategy equilibrium and all public-offers pure-strategy equilibria can be ranked in terms of the amount of trade. Since Theorem 1 establishes that every equilibrium with private offers has weakly more trade than any equilibrium with public offers, only the pure-strategy equilibrium with the most trade is a candidate for a private-offers equilibrium outcome.

We have shown that if equilibria differ in the two information structures, then there is more trade with private offers. Next, we show that equilibria with private and public offers do not always coincide. In particular, they must differ when the seller discounts future periods only a little. A high discount factor can alternatively be interpreted as frequent opportunities to trade as discussed in Section 4.1. We show that in this case, pure-strategy equilibria cease to exist with private offers.

THeORem 2. (i) With public offers, a pure-strategy equilibrium always exist.

(ii) With private offers, there exists a $\delta^{*}$ such that for all $\delta>\delta^{*}$ no pure-strategy equilibria exist.

Proof. (i) The existence of public-offer equilibria follows by backward induction. A buyer's expected period 2 profit conditional on his offer being accepted is given by

$$
\pi_{2}\left(k_{2} ; k_{1}\right)=\frac{1}{1-F\left(k_{1}\right)} \int_{k_{1}}^{k_{2}}(v(c)-\underbrace{\left((1-\delta) k_{2}+\delta v\left(k_{2}\right)\right)}_{p_{2}\left(k_{2}\right)}) f(c) d c .
$$


Since in equilibrium buyers must make zero profits (Lemma 2 ), any equilibrium cutoff of the continuation game $\kappa_{2}\left(k_{1}\right)$ must satisfy

$$
\pi_{2}\left(\kappa_{2}\left(k_{1}\right) ; k_{1}\right)=0 .
$$

Note that such a continuation cutoff $\kappa_{2}\left(k_{1}\right)$ always exists and is smaller than 1 because $v(1)=1$. So as to attract a cutoff type $k_{1}$ in the first period, buyers need to bid at least $p_{1}=(1-\delta) k_{1}+\delta p_{2}\left(\kappa_{2}\left(k_{1}\right)\right)$. Hence, buyers' profits in period 1 can be written as

$$
\pi_{1}\left(k_{1} ; 0\right)=\int_{0}^{k_{1}}(v(c)-\underbrace{\left((1-\delta) k_{1}+\delta p_{2}\left(\kappa_{2}\left(k_{1}\right)\right)\right)}_{p_{1}\left(k_{1}\right)} f(c) d c .
$$

Then

$$
k_{1}^{*}=\sup \left\{k \in[0,1] \mid \pi_{1}(k ; 0)>0\right\}
$$

(with $k_{1}^{*}=0$ if the set is empty) and $k_{2}^{*}=\kappa_{2}\left(k_{1}^{*}\right)$ supports an equilibrium. From now on we denote the equilibrium cutoffs in the game with public offers by $k_{t}^{*}$.

(ii) First, recall that period 1 buyers' profits with private offers

$$
\pi_{1}\left(k_{1} ; 0\right)=\int_{0}^{k_{1}}\left(v(c)-\left((1-\delta) k_{1}+\delta p_{2}\left(k_{2}^{*}\right)\right)\right) f(c) d c
$$

are differentiable in $k_{1}$ and the zero-profit condition

$$
\mathbb{E}\left[v(c) \mid c \in\left[0, k_{1}\right]\right]=p_{1}\left(k_{1}\right)
$$

must be satisfied for all $k_{1}$ in the support of the equilibrium strategy of period 1 buyers (Lemma 2). Similarly, profits must be equal to zero in period 2 and buyers must have correct beliefs about the period 1 cutoff.

Suppose the game with private offers has a pure-strategy equilibrium that induces the same cutoffs $k_{t}^{*}$ that we found in the game with public offers. Consider the incentives of buyers in the first period. With private offers, if buyers deviate to a higher price, to induce a marginally higher cutoff than $k_{1}^{*}$, we can compute using (5) that the net marginal benefit (NMB) of that deviation is

$$
\left.\frac{\partial}{\partial k_{1}} \pi_{1}\left(k_{1} ; 0\right)\right|_{k_{1}=k_{1}^{*}}=F\left(k_{1}^{*}\right) \cdot[\left.\frac{\partial}{\partial k_{1}} \mathbb{E}\left[v(c) \mid c \in\left[0, k_{1}\right]\right]\right|_{k_{1}=k_{1}^{*}}-\underbrace{\frac{\partial}{\partial k_{1}} p_{1}\left(k_{1}\right)}_{=1-\delta}] .
$$

Now, as $\delta \rightarrow 1$, it follows from the seller's indifference conditions that $k_{1}^{*} \rightarrow 0$. When we consider the limit $k_{1}^{*} \rightarrow 0$, we can apply l'Hopital's rule to obtain

$$
\left.\lim _{k_{1}^{*} \rightarrow 0} \frac{\partial}{\partial k_{1}} \mathbb{E}\left[v(c) \mid c \in\left[0, k_{1}\right]\right]\right|_{k=k_{1}^{*}}=\frac{v^{\prime}(0)}{2} .
$$

Thus,

$$
\left.\lim _{k_{1}^{*} \rightarrow 0} \frac{1}{F\left(k_{0}^{*}\right)} \cdot \frac{\partial}{\partial k_{1}} \pi_{1}\left(k_{1} ; 0\right)\right|_{k_{1}=k_{1}^{*}}=\frac{v^{\prime}(0)}{2}-(1-\delta)
$$


is strictly positive for large enough $\delta$ since $v^{\prime}(0)>0$. Thus, the NMB is positive if $k_{1}^{*}>0$, so the candidate pure-strategy equilibrium cannot be an equilibrium with private offers. If $k_{1}^{*}=0$, then this cannot be a private-offers equilibrium because any private-offers equilibrium must result in some trade in every period as we prove in Proposition 2. Hence, there exists a $\delta^{*}$ such that no pure-strategy equilibrium can be sustained with private offers for all $\delta>\delta^{*}$.

Intuitively, the difference in the two information structures can be seen as follows. With public offers, the seller has a stronger incentive to reject high price offers in period 1 than if the offer had been made privately: Suppose one of the buyers made an outof-equilibrium high offer. With public offers the seller gains additional reputation of her type being high by rejecting this offer, the strength of her signal being endogenously determined by the amount of money she left on the table. Consequently, her continuation value increases upon a rejection of the higher price. Instead, with private offers, she cannot use the out-of-equilibrium higher offer as a signal, so her continuation value remains constant. Thus, she has stronger incentives to accept the higher offer if it is private. Formally, this is reflected by different period 1 supply functions $p_{1}(k)$ in the two information structures. In particular, for all $k>k_{1}^{*}$, the price that makes $k$ indifferent with public offers is greater than the price with private offers:

$$
\underbrace{(1-\delta) k+\delta p_{2}\left(\kappa_{2}(k)\right)}_{p_{1}(k) \text { with public offers }}>\underbrace{(1-\delta) k+\delta p_{2}\left(k_{2}^{*}\right)}_{p_{1}(k) \text { with private offers }} .
$$

The effect is large enough to break down any potential pure-strategy equilibria with private offers if the discount factor is large enough because the seller's value of signaling to future buyers is higher if the next period is a very short time away. Thus, with high discount factors, if an equilibrium is to exist, buyers must make mixed price offers in both periods. ${ }^{16}$

\subsection{Distress and market breakdown}

Recall that we assumed that if the seller rejects offers at $t=2$, she captures $(1-\alpha)(v(c)-c)$ of the continuation surplus. The term $\alpha>0$ can be interpreted as a measure of distress. In the following discourse, we discuss how it affects equilibria in the two information regimes and show that for $\alpha>0$ and large enough discount factor, period 1 prices with private offers are higher than with public offers. Thus, privateoffers equilibria Pareto-dominate all public-offers equilibria, which concludes the proof of Theorem 1(iii) (a).

To this end, consider a game with public offers. We show that trade in period 1 can break down if $\alpha>0$ and $\delta$ is large. If some surplus is lost after the deadline, there is an

\footnotetext{
${ }^{16}$ If buyers did not mix in period 1 , then by regularity assumptions (1) and (2) there is only one cutoff that satisfies the zero-profit condition in period 2. Similarly, if buyers did not mix in period 2, then only one period 1 price would satisfy the period 1 zero-profit condition. Thus, if mixing occurs, it must occur in both periods.
} 
extra incentive to trade in period 2, right before the deadline. The higher is $\alpha$, the more types trade at the deadline. This leads to quiet period (i.e., no trade) in period 1 if $\delta$ is high. Formally, we make the following statement.

Proposition 1 (Quiet period). With public offers, for any $\alpha>0$ there exists a $\delta^{* *}<1$ such that if $\delta>\delta^{* *}$ in equilibrium, there is no trade in the first period.

Proof. In search of a contradiction, suppose there was trade in period 1 for arbitrarily large $\delta$ and let us denote the largest seller type trading in period 1 by $\hat{k}$. The highest price at which he could possibly be trading is $v(\hat{k})$ and as $\delta \rightarrow 1, \hat{k}$ converges to zero. Since the mass of types trading in period 2 is uniformly bounded from below for all $\delta$ if $\alpha>0$, the price at $t=2$ must be uniformly bounded away from $v(0)$. Thus, if $\delta$ is sufficiently close to 1 , for a type $-\hat{k}$ seller, the cost of waiting so as to trade at the higher price in the next period is negligible relative to the benefit, so she should not trade.

This logic can be extended to multiple periods as we show in the Appendix in Proposition 6 . The reason this logic does not apply when $\alpha=0$ is that in that case as $\delta$ increases to 1 , while probability of trade in period 2 is positive, it is not uniformly bounded away from zero. In fact, it converges to zero and the period 2 price converges to $v(\hat{k})$, and there can be trade in both periods along the sequence, as we have shown in the previous section. Thus, in contrast to HV who find that with public offers there is trade only in the first period, we find that without distress with public offers there is trade in every period and with distress there is no trade in the first period.

In contrast, with private offers, an equilibrium cannot have quiet periods (i.e., periods with zero probability of trade).

Proposition 2 (No quiet periods). With private offers, for all $\alpha$ and $\delta$ there must be a strictly positive probability of trade in every period.

Proof. Suppose that in the current period there was no trade but in the next period there would be some trade at a price $p$. The buyers could offer a price $p$ in the current period, attracting all sellers and some higher types that would have accepted $p$ in the next period. They accept because the offer is private and, thus, does not change the continuation game for the seller if she were to reject it. Such a deviation is profitable for buyers because buyers in the next period would have made nonpositive profit. Thus there cannot be quiet periods in equilibrium.

In particular, in period 1 there must be a positive probability of trade. This establishes another important difference in the equilibrium behavior across information structures. This difference allows us to easily argue that when $\alpha>0$ for high $\delta$, the opaque environment Pareto-dominates the transparent one and we conclude the proof of Theorem 1(iii) (a).

Proof of Theorem 1(iii)(a). We already know from Theorem 1(ii) that all types that sell in period 2 or wait until the deadline with public offers are better off with the private 
information structure. Proposition 1 implies that for $\alpha>0$ and $\delta$ sufficiently large, there are no sellers that trade in period 1 with public offers, while some types get to trade with private offers by Proposition 2. Thus, an equilibrium with private offers strictly Pareto-dominates any equilibrium with public offers, which concludes the proof of Theorem 1(iii) (a).

Remark 2. The reasoning in Proposition 1 and Proposition 2 does not rely on there being only two periods of trade. Hence, when $\alpha>0$, it is a very general result that there exists a $\delta^{*}<1$ such that for $\delta>\delta^{*}$, the opaque environment Pareto-dominates the transparent one.

\subsection{The linear and uniform case}

With linear valuation $v(c)=A c+B$ and $c$ being uniformly distributed on [0,1], we can fully characterize the set of private-offers equilibria and use this to show that the private information structure Pareto-dominates the public one. To this end, we first present a stronger version of Theorem 2 in the linear-uniform environment.

Theовем 3. Let $v(c)=A c+B$ be linear and let $c$ be uniformly distributed on $[0,1]$.

(i) With public offers, there is a unique equilibrium that is in pure strategies.

(ii) With private offers, there exists a

$$
\delta^{*}=1-\frac{A}{2} \in(0,1)
$$

such that the following statements hold:

(a) For all $\delta<\delta^{*}$, the equilibrium is unique and the equilibrium outcome coincides with the equilibrium outcome with public offers.

(b) For all $\delta>\delta^{*}$, no pure-strategy equilibria exist. Instead, there are multiple mixed-strategy equilibria. For any equilibrium with private offers, the expected price in the first period is strictly higher than the expected price with public offers.

Proof. (i) The unique public-offer equilibrium can be calculated using backward induction. In the continuation game starting in period 2 with the lowest seller type left being $k_{1}$, a buyer's expected profit conditional on his offer being accepted is given by

$$
\pi_{2}\left(k_{2} ; k_{1}\right)=\frac{k_{2}-k_{1}}{1-k_{1}}\left(\left(\frac{A}{2}-(1-(1-\alpha) \delta)-(1-\alpha) \delta A\right) k_{2}+(1-(1-\alpha) \delta) B+\frac{A}{2} k_{1}\right) .
$$

Thus, by the zero expected profit condition, the equilibrium period 2 cutoff in the continuation game is given by

$$
\kappa_{2}\left(k_{1}\right)=\frac{(1-(1-\alpha) \delta) B+\frac{A}{2} k_{1}}{1-(1-\alpha) \delta-\frac{A}{2}+(1-\alpha) \delta A} .
$$


Plugging this into the zero expected profit condition for period 1 ,

$$
\pi_{1}\left(k_{1} ; 0\right)=k_{1} \cdot\left(\left(\frac{A}{2}-(1-\delta)\right) k_{1}+B-\delta p_{2}\left(\kappa_{2}\left(k_{1}\right)\right)\right)=0,
$$

one can solve for the unique equilibrium period 1 cutoff. For $\alpha=0$ it is

$$
k_{1}^{*}=\frac{2 B \cdot(1-\delta)^{2}(2-A)}{2(1-\delta)(1-A)(A \delta-2 \delta+2)+A^{2}}
$$

and for $\alpha>0$ it can be calculated analogously. One caveat is that for

$$
1-\alpha<\frac{2(1-\delta)-A}{\delta(2-A-2 \delta-A(1-\delta))}
$$

the solution to the zero-profit condition can be negative, in which case $k_{1}^{*}=0$, which is a special case of Proposition 1. This pins down the equilibrium period 2 cutoff $k_{2}^{*}=$ $\kappa_{2}\left(k_{1}^{*}\right)$. For $\alpha=0$ it is given by

$$
k_{2}^{*}=\frac{2 B \cdot\left(2(1-\delta)^{2}+A \delta(1-\delta)\right)}{2(1-\delta)(1-A)(A \delta-2 \delta+2)+A^{2}} .
$$

Note that this is the unique solution of the cutoffs that jointly satisfy the zero-profit conditions of periods 1 and 2 .

(ii) However, the public-offers solution fails to be an equilibrium with private offers if buyers in period 1 have an incentive to deviate by increasing the price (and hence cutoff) slightly, i.e.,

$$
\left.\frac{\partial}{\partial k_{1}} \pi_{1}\left(k_{1} ; 0\right)\right|_{k_{1}=k_{1}^{*}}=k_{1}^{*} \cdot(\frac{A}{2}-\underbrace{(1-\delta)}_{\frac{\partial p_{1}}{\partial k_{1}}\left(k_{1}\right)})>0 .
$$

Importantly, the marginal benefit of increasing the price slightly with private offers is higher than with public offers because with public offers, the seller was more likely to reject such prices to signal to period 2 buyers. Thus private offers lead to more acceptance by higher types and possibly higher profits. The difference between the environments depends on the discount factor, since signaling is only attractive if the next offer will take place soon. Indeed, for high discount factors, $\delta>\delta^{*} \equiv 1-A / 2$, it is profitable for a buyer to deviate to higher prices (unless $k_{1}^{*}=0$ ). Consequently, there is no pure-strategy equilibrium with private offers if $\delta>\delta^{*}$ and $k_{1}^{*}>0$. Instead, if $\delta \leq \delta^{*}$, then buyers in period 1 do not have an incentive to deviate because their profit

$$
\pi_{1}\left(k_{1} ; 0\right)=k_{1} \cdot\left(\frac{A}{2} k_{1}+B-p_{1}\left(k_{1}\right)\right)
$$

is a quadratic function with a null at $k_{1}=0$ and $k_{1}=k_{1}^{*}$, and negative slope at $k_{1}^{*}$. Note that the public offers equilibrium results in no trade in period 1 (i.e., $k_{1}^{*}=0$ ) if and only if

$$
\delta>1-\frac{-1-(1-\alpha)+\sqrt{\alpha\left(1-(1-A)^{2}(1-\alpha)\right)}}{(2-A)(1-\alpha)},
$$


which is (7) solved for $\delta$. Since

$$
1-\frac{-1-(1-\alpha)+\sqrt{\alpha\left(1-(1-A)^{2}(1-\alpha)\right)}}{(2-A)(1-\alpha)}>1-\frac{A}{2}=\delta^{*},
$$

the public offers equilibrium outcome cannot be supported as an equilibrium with private offers if and only if $\delta>\delta^{*}$.

The discussion above establishes that if $\delta>\delta^{*}$, there can be only mixed-strategy equilibria in the game with private offers. Proposition 3 and Lemma 4 below together establish part (ii) (b) of Theorem 3. The proposition summarizes properties that all mixedstrategy equilibria must satisfy and the lemma establishes existence.

Proposition 3. Suppose $\delta>\delta^{*}=1-A / 2$. In any mixed-strategy equilibrium with private offers, the following statements hold:

(i) In period 2, buyers mix between exactly two prices, which result in the two cutoffs given by

$$
\underline{k}_{2}=\frac{B(1-(1-\alpha) \delta)}{A(1-\alpha) \delta-(1-\alpha) \delta+1-\frac{A}{2}}, \quad \bar{k}_{2}=\frac{B\left(1-(1-\alpha) \delta^{2}\right)}{A(1-\alpha) \delta^{2}-(1-\alpha) \delta^{2}+1-\frac{A}{2}},
$$

where $\underline{k}_{2}$ is chosen with probability

$$
q_{2} \equiv \frac{\frac{A}{2}-(1-\delta)}{\delta(A(1-\alpha) \delta+1-(1-\alpha) \delta)} .
$$

(ii) In period 1, buyers mix between prices that induce cutoffs 0 and cutoffs that lie in $\left(\underline{k}_{2}, \bar{k}_{2}\right)$. Cutoff 0 is induced on the equilibrium path with a positive probability.

For the proof, see the Appendix.

It only remains to show that a mixed equilibrium exists:

LeMмa 4. For $\delta>\delta^{*}$, a mixed-strategy equilibrium exists in which buyers in period 1 mix between exactly two prices.

See the Appendix for the proof.

This concludes the proof of Theorem 3(ii)(b). The proof of Theorem 3(ii)(a) can be found in the Appendix. It is proven jointly with Proposition 3.

How does the mixing help resolve the problem of nonexistence of equilibrium? Consider any cutoff $k_{1}>0$ consistent with the equilibrium outcome in period 1 . It must be that $\pi_{1}(k ; 0)=0$ and $\partial \pi_{1}(k ; 0) / \partial k \leq 0$ at that cutoff. As we argued above, the sign of $\partial \pi_{1}(k ; 0) / \partial k$ depends on the sign of $\partial\left(\mathbb{E}[v(c) \mid c \leq k]-p_{1}(k)\right) / \partial k$. Mixing in period 2 changes the derivative of $p_{1}(k)$. In particular, if $k_{1}$ trades in period 2 if the price offer is high and does not trade in period 2 if the offer is low, then $p_{1}(k)=$ $\delta \mathbb{E}\left[\max \left\{p_{2}(\tilde{k}), p_{2}(k)\right\}\right]+(1-\delta) k$, where $p_{2}(k)$ (defined in (4)) is the seller's continuation payoff if she rejects period 2 prices and $\tilde{k}$ is the equilibrium period 2 cutoff distributed 


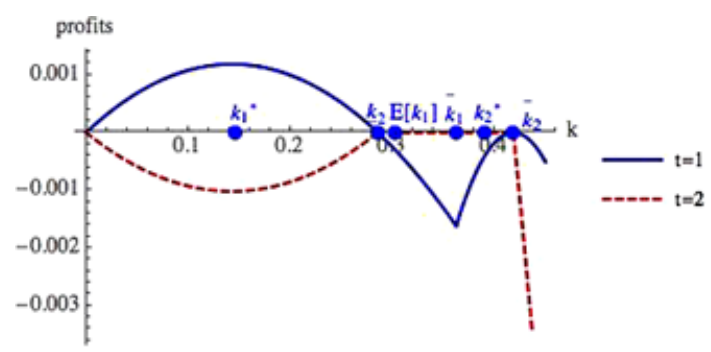

Figure 1. A buyer's equilibrium profit in periods 1 and 2 conditional on acceptance as a function of the marginal seller type $k$.

according to $K_{2}$. Mixing in period 2 makes the seller's continuation payoff in period 1 more sensitive to her type and, hence, the supply function $p_{1}(k)$ becomes less elastic. If the probability of $k_{1}$ not trading in period 2 is high enough, then period 1 buyers have no incentive to increase prices.

In equilibrium buyers must mix over period 1 offers for two reasons. First, if the posterior belief in period 2 were a truncation of the uniform prior, there would be a unique continuation equilibrium price. Mixing in period 1 is needed to induce a posterior such that mixing in period 2 is indeed a continuation equilibrium. Second, and more generally, note that the lowest type in the support of $F_{2}$ trades in period 2 for sure (recall $v(c)>c)$. If the lowest cutoff induced in period 1 were strictly positive, then for that type $\partial p_{1}(k) / \partial k=1-\delta$. As discussed above, that would imply $\partial \pi_{1}(k ; 0) / \partial k>0$ for $\delta>\delta^{*}$ and buyers would have a profitable deviation. Therefore in equilibrium, buyers in period 1 must make with positive probability a nonoffer, i.e., offer a low price that is rejected by all types. ${ }^{17}$ At the same time, it cannot be that no type trades in period 1 . If so, buyers could deviate to the highest price offered in equilibrium in period 2 and make a strictly positive profit (since that price would be accepted by types better than those that trade in period 2).

In the proof of Theorem 3, we construct an equilibrium in which buyers in period 1 mix between exactly two price offers. Figure 1 illustrates the profits of a buyer in periods 1 and 2 conditional on his offer being accepted. The figure also contains the equilibrium cutoffs with private and public offers in periods 1 and 2 . First, it is worth noting that with private offers, since $\pi_{1}$ is nonpositive for all $k$, buyers cannot make positive profits even conditional on acceptance and even assuming the realized offer of the other buyer is 0 . Thus, buyers are indifferent between trading with no seller and making offers acceptable by types between $\left[\underline{k}_{2}, \bar{k}_{2}\right]$. Second, note that $\pi_{2}$ is only positive below $\underline{k}_{2}$ but in equilibrium, since the other buyer is mixing between $\underline{k}_{2}$ and $\bar{k}_{2}$, those would be losing offers and thus effectively $\pi_{2}$ is never positive. In the particular equilibrium that we characterize, period 1 buyers mix between a non-offer, resulting in cutoff 0 , and a high offer, resulting in cutoff $\bar{k}_{1}$. Moreover, one can see that the expected period 1 cutoff $\mathbb{E}\left[k_{1}\right]$ with

\footnotetext{
${ }^{17}$ In equilibrium the lowest on-path period 1 cutoff is $k_{1}=0$. While at that cutoff $\partial(\mathbb{E}[v(c) \mid c \leq k]-$ $\left.p_{1}(k)\right) / \partial k>0$, the reservation prices of the low types are sufficiently high so that for all cutoffs $k \in\left(0, \underline{k}_{2}\right)$, $\pi_{1}(k ; 0)<0$. In particular, $p_{1}(0)>v(0)$.
} 
private offers is significantly higher than the cutoff type with public offers $k_{1}^{*}$, i.e., in period 1, more trade takes place in expectation with private offers. We show in the proof of Theorem 1(iii)(b) that this observation is more general.

Even though the private-offers equilibrium strategy in period 1 is not unique, all equilibrium strategies have some properties in common. In particular, the expected cutoff type is constant across equilibria and on average higher than with public offers. This allows us to conclude the proof of Theorem 1(iii)(b).

Proof of Theorem 1(iii)(b). The following lemma first establishes that the expected period 1 cutoff is constant across equilibria with private offers. Thus, we can simply calculate the expected period 1 cutoff with private offers and show that it is greater than $k_{1}^{*}$. The formal proof of this lemma can be found in the Appendix.

Lemma 5. If $\delta>\delta^{*}$, the following statements hold:

(i) The expected cutoff in period 1 is constant across all equilibria with private offers.

(ii) Denoting the expected equilibrium cutoff in period 1 with private offers by $\mathbb{E}^{K_{1}}\left[k_{1}\right]$, it is higher than the equilibrium cutoff with public offers:

$$
\mathbb{E}^{K_{1}}\left[k_{1}\right]>k_{1}^{*} \text {. }
$$

Hence, if the equilibrium distribution of period 2 cutoffs is given by $K_{2}$, the reserve price of any type that trades in period 1 is at least

$$
p_{1}(k) \geq(1-\delta) k+\delta \mathbb{E}^{K_{2}}\left[p_{2}\left(k_{2}\right)\right]
$$

because that type has the option not to sell in period 2. Integrating the reserve prices over the equilibrium distribution of the period 1 cutoff types, we get the average transaction price in period $1,{ }^{18}$

$$
\mathbb{E}^{\operatorname{Private}}\left[p_{1}\right] \equiv \int_{0}^{1} p_{1}(\tilde{k}) d K_{1}(\tilde{k}) \geq(1-\delta) \underbrace{\int_{0}^{1} \tilde{k} d K_{1}(\tilde{k})}_{>k_{1}^{*}}+\delta \underbrace{\mathbb{E}^{K_{2}}\left[p_{2}\left(k_{2}\right)\right]}_{>p_{2}\left(k_{2}^{*}\right)},
$$

where $K_{1}$ is the cdf of the equilibrium distribution of period 1 cutoffs with private offers. This is greater than the period 1 public-offer price $p_{1}\left(k_{1}^{*}\right)=(1-\delta) k_{1}^{*}+\delta p_{2}\left(k_{2}^{*}\right)$ because the average cutoff in period 1 is higher and the average price in period 2 is also higher (as we showed in Theorem 1(i)). Thus, all seller types $c<k_{1}^{*}$ are better off with private offers: they either sell in the first period at a higher expected price or choose to sell in the second period, which must give them higher profits by revealed preference. Consequently, we have established the Pareto ranking of equilibria, i.e., all seller types are ex ante better off with the private-information structure than with the public-information structure. This concludes the proof of Theorem 1.

\footnotetext{
${ }^{18}$ Recall that in the private-offers equilibrium buyers make a non-offer with positive probability. That price is unbounded from below, but the equilibrium payoffs of all types can be computed as if the price offered in that case was equal to the reserve price of the lowest type, as we do in this expression.
} 


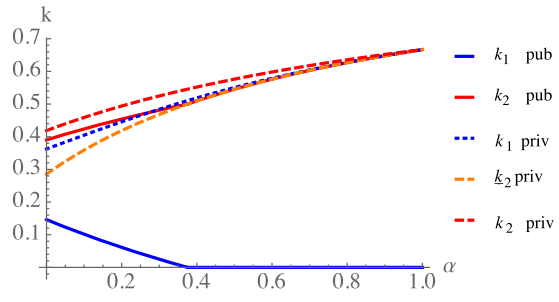

(a) Cutoffs for $\delta=0.8, A=B=0.5$

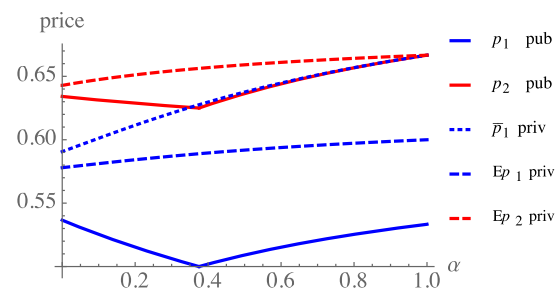

(b) Expected prices for $\delta=0.8, A=B=0.5$

Figure 2. Role of distress.

For the uniform-linear case with $A=B=0.5$ and $\delta=0.8$, we can calculate equilibria in which buyers mix between exactly two prices in every period for different levels of distress. The resulting cutoffs and prices as a function of the level of distress $\alpha$ are illustrated in Figure 2. ${ }^{19}$

Indeed, there is more trade the more distress is faced at the deadline (i.e., as $\alpha$ increases). However, trade breaks down in period 1 with high level of distress (i.e., $\alpha$ greater than $\approx 0.37$ ) if offers are public. In contrast, this effect is almost completely alleviated with private offers. Hence, if distress is a severe issue, the benefit of opaque environments is potentially even higher than without distress at the deadline. Note that the total level of trade is the same when $\alpha=1$, but with private offers most of the trade occurs in period 1 . In contrast, if $\alpha<1$, total expected trade is also higher with private offers.

\section{Robustness and generalizations}

\subsection{More than two periods}

We now generalize Theorem 2 by allowing more opportunities to trade before the deadline, with each period $\{0, \Delta, \ldots, 1-\Delta\}$ having length $\Delta$ and the discount rate being $r$, i.e., $\delta=e^{-r \Delta}$.

The proof of Lemma 3 can be extended by induction to show that an inverse supply function exists in every period, even with more opportunities to trade, although it does not have to be defined on the entire cutoff space. The following analysis is independent of what happens at the deadline and therefore holds true for all $\alpha \in[0,1]$.

The existence of a pure-strategy equilibrium with public offers can be shown by construction in two steps. First, we define for each period a mapping that maps today's cutoff to tomorrow's cutoff by backward induction. Then the cutoff in the first period is this function realized at cutoff zero and all other cutoffs can be inductively found (for formal construction, see the proof of Theorem 4 in the Appendix). In general, multiple purestrategy equilibria can coexist. To illustrate the dynamics in pure-strategy equilibria, we have plotted in Figure 3 prices at which different seller types trade for $v(c)=0.5+0.5 c$,

\footnotetext{
${ }^{19}$ Note that in the case of non-offers we assume that prices are offered that make the zero-type seller just indifferent between accepting and rejecting (instead of assuming the price is zero). We plot these prices since they were used for the welfare comparison.
} 


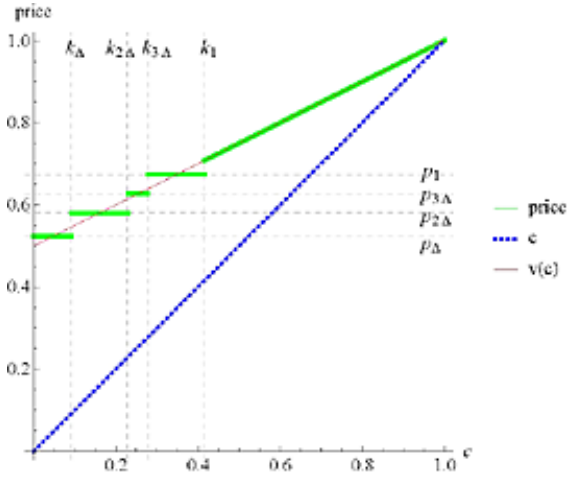

(a) Prices as a function of seller types for $\Delta=\frac{1}{4}$

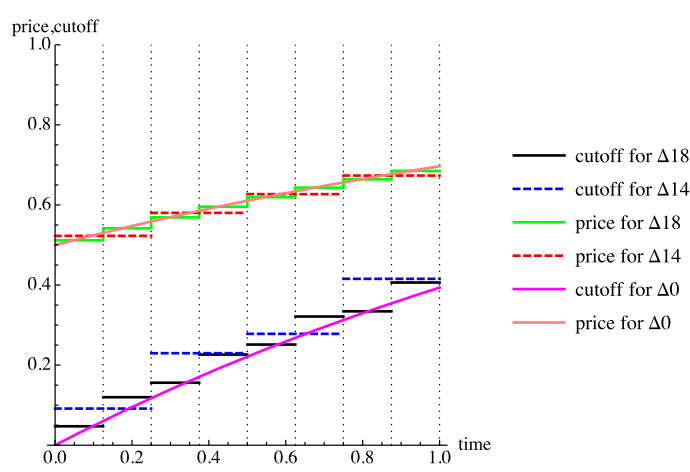

(b) Prices and cutoffs over time for $\Delta \in\left\{\frac{1}{4}, \frac{1}{8}\right\}$

FIGURE 3. Pure-strategy equilibria with $r=0.5$ and $v(c)=(c+1) / 2$.

$r=0.5$, and $\Delta \in\left\{\frac{1}{4}, \frac{1}{8}\right\}$ as well as $\Delta \rightarrow 0$. The term $p_{t}$ denotes the price and $k_{t}$ denotes the equilibrium cutoff in period $t$.

Theorem 4 fully generalizes Theorem 2 .

THEOREм 4. (i) With public offers, there exists a pure-strategy equilibrium for all $0<\delta<1$.

(ii) Equilibrium cutoffs (and prices) in any pure-strategy equilibrium with private offers correspond to equilibrium cutoffs (and prices) in a pure-strategy equilibrium with public offers.

(iii) There exists $a \Delta^{*}<1$ such that if $\Delta<\Delta^{*}$, there is no pure-strategy equilibrium with private offers.

Proof. Here we only present the proofs of (ii) and (iii). Part (i) was intuitively discussed above and is formally proven in the Appendix.

With private offers, there must be trade with positive probability in each period because buyers can always mimic the strategies of future buyers as we have already discussed in Proposition 2. Hence, in any pure-strategy equilibrium with cutoffs $\left(k_{\Delta}^{*}, \ldots, k_{1-\Delta}^{*}\right)$, the zero-profit condition

$$
\mathbb{E}\left[v(c) \mid c \in\left[k_{t-\Delta}^{*}, k_{t}^{*}\right]\right]=p_{t}\left(k_{t}^{*}\right)
$$

must be satisfied for all $t$. Moreover, a buyer's expected period 1 profit conditional on having the higher bid is given by

$$
\pi_{0}(k ; 0)=F(k) \cdot \underbrace{\left[\mathbb{E}[v(c) \mid c \in[0, k]]-p_{0}(k)\right]}_{=0 \text { at } k=k_{0}^{*}} .
$$


Analogously to Theorem 2, one can show that the NMB of a deviation is given by

$$
\left.\frac{\partial}{\partial k} \pi_{0}(k ; 0)\right|_{k=k_{0}^{*}}=F\left(k_{0}^{*}\right) \cdot[\left.\frac{\partial}{\partial k} \mathbb{E}[v(c) \mid c \in[0, k]]\right|_{k=k_{0}^{*}}-\underbrace{\frac{\partial}{\partial k} p_{0}(k)}_{1-\delta}] .
$$

By the same argument we used to provide intuition for Theorem 2, it follows that for large $\delta$ (i.e., small $\Delta$ ), period 1 buyers can profitably deviate by offering a higher price, establishing part (iii) of Theorem 4.

Finally, note that in any pure-strategy equilibrium with private offers, buyers must have correct beliefs about the cutoff type of the seller. As the seller is more inclined to reject higher price offers with public offers, any pure-strategy equilibrium with private offers must correspond to a pure-strategy equilibrium with public offers. This concludes the proof of Theorem 4(ii).

\subsection{Gap at the top $v(1)>1$}

Throughout the paper, we have assumed that $v(1)=1$. This assumption together with continuity and monotonicity of $v(c)$ guarantees that in any equilibrium, a positive mass of high type sellers do not trade before the deadline. The reason is that the expected value of buyers is always smaller than 1 , so that the highest type $c=1$ never trades before information is revealed. Hence, we did not have to worry about off-equilibrium beliefs of buyers if they see a seller rejecting even though on the equilibrium path, all sellers should have traded. The freedom in choice of off-equilibrium beliefs could lead to additional multiplicities of equilibria.

Nevertheless, all results can easily be generalized to settings with $v(1)>1$ if we assume that the lemons problem is severe enough so that trade does not end before the deadline or if we make some out-of-equilibrium belief assumptions. For example, if buyers' beliefs remain unchanged (or become more pessimistic) after the last period of trade, the game can still be solved by backward induction and the same arguments can be applied as in the proofs of the theorems.

\subsection{No gap at the bottom $(v(0)=0)$}

We made the assumption that $v(0)>0$ to make sure that there is always some trade before time 1 . If we have no gap at the bottom, then it is possible for trade to completely unravel in all periods if the lemons condition is satisfied. For $v(c)=A c+B$, the lemons condition is $(A+B) / 2<1$.

Proposition 4. If $B=0$ and $A<2$, there always exists a pure-strategy equilibrium with private and public offers. In that equilibrium, there is no trade before the deadline.

\subsection{Two types}

Several of the recent papers that look at dynamic adverse selection consider only two possible types (e.g., Daley and Green 2012, Camargo and Lester 2014, and 
Nöldeke and Van Damme 1990) or use the two type case as examples (e.g., Swinkels 1999, HV, and Deneckere and Liang 2006). Hence it is interesting to explore equilibria of our games with two types $H$ and $L$. So as to be closer to the HV setup, we assume there is no opportunity of trade after the last period (i.e., $\alpha=1$ ).

Consider a situation with $v_{H} \geq c_{H}$ and $v_{L}>c_{L}=0$, where the seller's cost is $c_{L}$ with probability $\phi$. Let $\phi$ satisfy the static lemons condition:

$$
\phi v_{L}+(1-\phi) v_{H}<c_{H}
$$

Then we show that equilibria in both information structures coincide.

Proposition 5. With two seller types and two opportunities to trade, equilibria with private and public offers coincide for every $\delta$.

This points out that in a dynamic setup it can be important to have a rich enough type space. The differences in results are driven by the inability of sellers to have a rich signal space because Bertrand competition only allows for two prices that make one of the two seller types indifferent between selling and waiting. The formal analysis can be found in the Appendix.

\section{Conclusion}

In this paper we have illustrated the "continuation" effect of information disclosure, that hiding past offers results in more trade. While we have illustrated it in a model with one asset and interdependent values, the same economic reasoning applies to other setups. For example, a seller of multiple units on eBay who has private information about the value or cost of those goods, is likely to have a less elastic supply if the past transaction prices are public than if they are private. Indeed eBay in 2013 changed its policy from displaying past accepted price offers (below asking price) to hiding the amount and only revealing that an offer had been accepted-a change we would expect to lead to more efficient trade.

As argued long ago by Hayek (1945), prices can be valuable to other members of the economy who use the information contained in market prices to make decisions. In more recent work, Asriyan et al. (2015) provide a different argument in favor of transaction transparency. They show that endogenous information in transaction prices, if made publicly available, leads to the existence of equilibria that Pareto-dominate opaque markets in which transaction prices are not public information. The findings in our paper suggest that although transparently reporting actual transaction prices might be beneficial, it is important to have opaqueness of rejected offers, in particular when trade takes place frequently. Our analysis also shows that when thinking about policy and how to best resolve these trade-offs the details of the market structure, such as the timing of competition, and the level of distress are likely to play an important role. 
Apendix

\section{Proofs: Model and preliminaries}

Proof of Lemma 1. In both information structures, the seller accepts a price $p_{2}$ if and only if $p_{2}-c$ is greater than $\delta \cdot(1-\alpha)(v(c)-c)$. Since $\delta(1-\alpha)<1$, if a seller type $c$ weakly prefers to accept $p_{2}$, then all types $c^{\prime}<c$ strictly prefer to accept it. Similarly, in period $1, p_{1}$ is accepted by the seller if and only if $p_{1}-c$ is higher than the payoff from accepting the period 2 price given by $\delta \cdot\left(\mathbb{E}\left[p_{2}\right]-c\right)$ and is higher than waiting for the deadline $\delta^{2}(1-\alpha)(v(c)-c)$. The price $p_{2}$ is independent of $c$. Since the derivative of the continuation payoff with respect to $c$ is less than 1, again if a seller type $c$ prefers to accepts $p_{1}$, then all types $c^{\prime}<c$ strictly prefer to accept it. (Note that it is irrelevant that, with public offers, $p_{2}$ is a function of $p_{1}$.)

Generalization of this logic to more than two periods is straightforward.

Proof of Lemmas 2 and 3. (i) Private offers: With private offers, beliefs of buyers are independent of price histories. Hence, the continuation game in an equilibrium is unaffected by past offers. We argue by backward induction.

In period 2, a seller of type $c$ accepts an offer $p$ if and only if $p \geq(1-\alpha) \delta v(c)+$ $(1-(1-\alpha) \delta) c \equiv p_{2}(c)$. The price $p_{2}$ is increasing and continuous. Consequently, $p_{2}(k)=k_{2}^{-1}(k)$ is the unique price that results in a cutoff $k$ in period 2 .

In period 1 , the continuation payoff of a seller $c$ who rejects is given by

$$
\begin{aligned}
W(c) & =\delta \cdot \int_{c}^{1}\left(p_{2}(\tilde{k})-c\right) d K_{2}(\tilde{k})+\delta^{2} \cdot K_{2}(c) \cdot(1-\alpha) \cdot(v(c)-c)+c \\
& =\delta\left[\left(\int_{c}^{1} p_{2}(\tilde{k}) d K_{2}(\tilde{k})\right)+K_{2}(c) p_{2}(c)\right]+(1-\delta) c .
\end{aligned}
$$

Since $p_{2}$ is increasing, $W(c)$ is increasing and continuous. The equality $p_{1}(c)=W(c)$ defines the inverse supply function. Note that $p_{t}(k)$ is the unique price that results in a cutoff $k$ and hence $p_{t}=k_{t}^{-1}$.

An analogous argument with backward induction can be made for more than two periods.

In period 2, the profits must be zero because buyers' continuation profits are continuous in period 2 prices (and cutoffs). Since in equilibrium, period 2 prices are not affected by period 1 prices, period 1 profits are also continuous in period 1 prices and, hence, the zero-profit condition must hold.

(ii) Public offers: With public offers, it follows analogously to the private-offers case that $p_{2}(k)=\delta(1-\alpha) v(k)+(1-\delta(1-\alpha)) k$. Hence, period 2 profits of buyers (conditional on offering the highest price) are given by

$$
\pi_{2}\left(k ; k_{1}\right)=\frac{1}{1-F\left(k_{1}\right)} \cdot \int_{k_{1}}^{k}\left(v(c)-p_{2}(k)\right) f(c) d c,
$$

which is continuous. Hence, by the Bertrand-competition logic, any period 2 cutoff in a continuation equilibrium $\kappa_{2}\left(k_{1}\right)$ must satisfy the zero-profit condition and for all 
$k>\kappa_{2}\left(k_{1}\right)$ it must hold that $\pi_{2}\left(k ; k_{1}\right) \leq 0$. Moreover, the assumption that (1) is decreasing and (2) guarantees that $\kappa_{2}\left(k_{1}\right)$ is unique because the assumption implies that the first order condition defining $\kappa_{2}\left(k_{1}\right)$, which can be written as

$$
F(c) \cdot(1-(1-\alpha) \delta) \cdot\left[\frac{f(c)}{F(c)}(v(c)-c)-\left(\frac{(1-\alpha) \delta v^{\prime}(c)}{1-(1-\alpha) \delta}+1\right) \cdot\left(1-\frac{F\left(k_{1}\right)}{F(c)}\right)\right]=0,
$$

has a unique solution. Thus, by continuity of $\pi_{2}(\cdot ; \cdot), \kappa_{2}(\cdot)$ must be continuous. By the zero-profit condition, for $k_{1}>k_{1}^{\prime}, \kappa_{2}\left(k_{1}\right) \geq \kappa_{2}\left(k_{1}^{\prime}\right)$ because for all $k \geq \kappa_{2}\left(k_{1}\right), \pi_{2}\left(k ; k_{1}\right) \leq$ 0 and, therefore, the same must hold for all $k_{1}>k_{1}^{\prime}$. Hence, $\kappa_{2}(\cdot)$ must be increasing and

$$
p_{1}(k)=\delta \cdot k+(1-\delta) \cdot p_{2}\left(\kappa_{2}(k)\right)
$$

is increasing, well defined, and continuous. Finally, the zero-profit condition must be satisfied in period 1 because profits of buyers

$$
\pi_{1}(k ; 0)=\int_{0}^{k}\left(v(c)-p_{1}(k)\right) f(c) d c
$$

are continuous in $k$.

\section{Proofs: Distress, transparency, and welfare}

Proof of Proposition 3 and Theorem 3(ii)(a). Before we do the actual construction of equilibrium cutoffs, we need to show some properties about the type of mixing that can occur in an equilibrium. In Lemmas 7 and 8 we show that for $\delta<\delta^{*}$, buyers at most mix between countably many prices and that with $\delta>\delta^{*}$, there is only countable mixing after the first period. So as to prove these statements, the following lemma is useful.

Lemma 6. With private offers, $p_{1}(k)$ is differentiable almost everywhere and differentiable from the right everywhere. The derivative

$$
\frac{\partial}{\partial k} p_{1}(k)=1-\delta\left(1-K_{2}(k)\right)-(1-\alpha) \delta^{2} K_{2}(k)(1-A)(>0)
$$

is nondecreasing.

The proof of this lemma follows immediately from the fact that $K_{2}$ is a cdf. It is worth noting that this lemma generalizes to a multiperiod setup by induction.

Lemma 7. If $\delta<1-A / 2$, buyers in period 1 mix at most between countably many cutoffs. If $\delta>1-A / 2$ and expected period 1 profit $\pi_{1}(k ; 0)=0$ for all $k \in(a, b)$, then any $k \in(a, b)$ cannot be in the support of $K_{2}$ since it must hold that

$$
K_{2}(k)=\frac{\delta-1+\frac{A}{2}}{\delta \frac{\partial}{\partial k} p_{2}(k)} .
$$


Proof. In period 1, expected buyers' profits are given by

$$
\pi_{1}(k ; 0)=k \cdot\left[\frac{A}{2} k+B-p_{1}(k)\right] .
$$

If buyers mix between all cutoffs $k \in(a, b)$ at time 0 , then they must make zero profits for all such cutoffs, i.e., for all $k \in(a, b)$,

$$
\delta\left(\int_{k}^{1} p_{2}(\tilde{k}) d K_{2}(\tilde{k})+K_{2}(k) p_{2}(k)\right)+k(1-\delta)=\frac{A}{2} k+B
$$

or, equivalently,

$$
\delta\left(\int_{k}^{1} p_{2}(\tilde{k}) d K_{2}(\tilde{k})+K_{2}(k) p_{2}(k)\right)=\left(\delta-\left(1-\frac{A}{2}\right)\right) k+B .
$$

Note that the left hand side of the identity must be nondecreasing in $k$, so if $\delta<1-A / 2$, then there cannot be mixing on $(a, b)$ in the first period. If $\delta \geq 1-A / 2$, then the left hand side is differentiable, so the right hand side must be differentiable, so that

$$
K_{2}(k)=\frac{\delta-\left(1-\frac{A}{2}\right)}{\delta \frac{\partial}{\partial k} p_{2}(k)}
$$

on $k \in(a, b)$. Since $K_{2}$ is a cdf, $\frac{\partial}{\partial k} p_{2}(k)$ cannot be increasing on $(a, b)$, so that by Lemma $6, \frac{\partial}{\partial k} p_{2}(k)$ must be constant on $(a, b)$. This implies that the support of $K_{2}$ is disjoint from $(a, b)$.

LEMма 8. With private offers and if $\delta>\delta^{*}$, all mixed-strategy equilibria must satisfy the following properties.

(i) In period 2, buyers mix between at most countably many prices.

(ii) If buyers in period 1 mix continuously between prices that result in cutoffs in an interval $(a, b)$, then buyers in periods 2 never choose a price that results in a cutoff in $(a, b)$.

Proof. Assume there exists an interval $(a, b)$ such that buyers in period 2 mix between all cutoffs, i.e., for all $k \in(a, b)$,

$$
\pi_{2}\left(k ; K_{1}\right)=\int_{0}^{k} \int_{0}^{c} \frac{1}{1-\tilde{k}} d K_{1}(\tilde{k})\left(A c+B-p_{2}(k)\right) d c=0 .
$$

After applying integration by parts and setting

$$
H(k) \equiv \int_{0}^{k}\left(\int_{0}^{c} \int_{0}^{x} \frac{1}{1-\tilde{k}} d K_{1}(\tilde{k}) d x\right) d c
$$

one can see that this is equivalent to the ordinal differential equation

$$
A H^{\prime}(k) k-A H(k)=H^{\prime}(k)\left(p_{2}(k)-B\right) .
$$


Thus, we can conclude that

$$
H(k) \equiv \int_{0}^{k}\left(\int_{0}^{c} \int_{0}^{x} \frac{1}{1-\tilde{k}} d K_{1}(\tilde{k}) d x\right) d c=\text { const } \cdot \exp \left(\int_{0}^{k} \frac{1}{z-\frac{p_{2}(z)-B}{A}} d z\right)
$$

and by Fubini's theorem,

$$
H(k)=\int_{0}^{k} \frac{k-\tilde{k}}{2(1-\tilde{k})} d K_{1}(\tilde{k})
$$

which is increasing because $(k-\tilde{k}) /(2(1-\tilde{k}))>0$ for $0<\tilde{k}<k$. Thus, the cdf $K_{1}(\cdot)$ must be strictly increasing everywhere on $(a, b)$. Hence, if buyers mix on $(a, b)$ in period 2 , then they must mix in period 1 , which is a contradiction by Lemma 7 . Hence, there cannot be mixing on an interval in period 2 .

Now, the proof of Proposition 3 follows in three steps. First, we show in Step 1 that buyers in period 2 mix between exactly two prices and we show the first part of (ii). Step 2 discusses the second part of (ii), i.e., that there must be non-offers with positive probability in period 1. Finally, in Step 3 we can pin down the exact values of $\underline{k}_{2}$ and $\bar{k}_{2}$.

STEP 1. In any mixed-strategy equilibrium with private offers, period 2 buyers mix between exactly two prices resulting in cutoffs $\underline{k}_{2}$ and $\bar{k}_{2}$, and period 1 cutoffs must be in $\{0\} \cup\left[\underline{k}_{2}, \bar{k}_{2}\right]$.

First, note that buyers in period 1 must mix between at least two cutoffs. The reason is that if buyers in period 1 were to play pure strategies, then there is a unique price at which period 2 buyers make zero profits, i.e., the unique Bertrand equilibrium in that period contains only pure strategies of the buyers.

Consider the continuation game in period 2 given beliefs about the current cutoffs represented by the cdf $K_{1}$. Buyers' profits are then given by

$$
\begin{aligned}
& \pi_{2}\left(k_{2} ; K_{1}\right) \\
& \quad=\int_{0}^{k_{2}}\left(\int_{0}^{c} \frac{1}{1-\tilde{k}} d K_{1}(\tilde{k})\right)\left(A c+(1-(1-\alpha) \delta) B-k_{2}((1-\alpha) \delta A+1-(1-\alpha) \delta)\right) d c .
\end{aligned}
$$

The term $\pi_{2}$ is continuous and at the smallest element $k_{1}<1$ in the support of $K_{1}$, for all $\epsilon$ small enough, we have

$$
\begin{aligned}
& \left.\frac{\partial \pi_{2}}{\partial k_{2}}\left(k_{2} ; K_{1}\right)\right|_{k_{2}=k_{1}+\epsilon} \\
& =\int_{0}^{k_{1}+\epsilon} \frac{1}{1-\tilde{k}} d K_{1}(\tilde{k})(1-(1-\alpha) \delta)\left(B+\left(k_{1}+\epsilon\right)(A-1)\right) \\
& \quad-\int_{k_{1}}^{k_{1}+\epsilon}\left(\int_{0}^{c} \frac{1}{1-\tilde{k}} d K_{0}(\tilde{k})\right) d c(A(1-\alpha) \delta+1-(1-\alpha) \delta)
\end{aligned}
$$




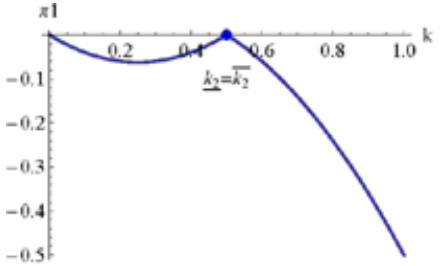

(a) Mixing between two cutoffs

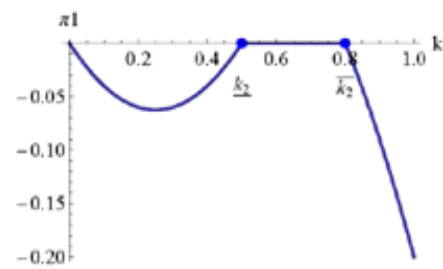

(b) Mixing on an interval

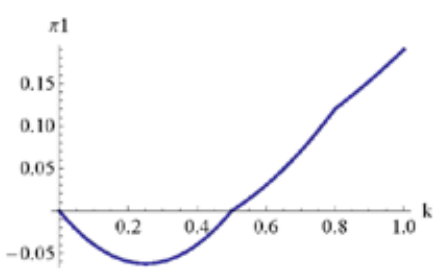

(c) No mixing

FIgURE 4. Possible shapes of buyers' profits in period 1.

$$
\begin{aligned}
>\int_{0}^{k_{1}+\epsilon} \frac{1}{1-\tilde{k}} d K_{1}(\tilde{k}) \\
\\
\quad \cdot\left[(1-(1-\alpha) \delta)(B+k_{1} \underbrace{(A-1)}_{=-B})-\epsilon(2(A(1-\alpha) \delta+1-(1-\alpha) \delta)-A)\right]
\end{aligned}
$$

$>0$,

so in equilibrium, buyers in period 2 do not choose prices that result in a cutoff type smaller than or equal to $k_{1}$ with positive probability since if they did, increasing the price a little bit would be a profitable deviation for any buyer. In particular, in any equilibrium, seller types close to zero trade in period 2, so that $K_{2}\left(k_{2}\right)=0$ for small $k_{2}$.

By Proposition 8, the support of $K_{2}$ is discrete and $p_{1}(\cdot)$ is piecewise linear and continuous, and by Lemma 6 , it is also weakly convex. Hence, buyers' expected profit in period 1 ,

$$
\pi_{1}(k ; 0)=k \cdot(\frac{A}{2} k+B-\underbrace{\left.\left(\delta\left[\left(\int_{k}^{0} p_{2}(\tilde{k}) d K_{2}(\tilde{k})\right)+K_{2}(k) p_{2}(k)\right]+k(1-\delta)\right)\right)}_{p_{1}(k)},
$$

is continuous, piecewise quadratic, and at any cutoff in the support of $K_{2}$ it has a "downward" kink (that is, the slope is dropping discontinuously) because of the convexity of $p_{1}$ (Lemma 6). Hence, in any mixed-strategy equilibrium, expected period 1 profits must qualitatively look like one of the graphs in Figure 4.

This allows us to conclude that for $\delta<1-A / 2$, there cannot be mixed-strategy equilibria as in that case, profits are increasing at 0 , so there cannot be mixing in period 1 . This concludes the proof of Theorem 3(ii) (a).

For small $k, p_{1}(k)=\delta \int_{k}^{1} p_{2}(\tilde{k}) d K_{2}(\tilde{k})+k(1-\delta)$ because $K_{2}(k)=0$ for small $k$. Hence, the left-most parabola must be open above if $A / 2-(1-\delta)>0$. Since trade cannot terminate before the deadline in equilibrium, we can exclude the possibility of the expected profit function in period 1 having a shape as in Figure 4(c). Hence, there exist cutoffs $0<\underline{k}_{2} \leq \bar{k}_{2}<1$ such that period 1 buyers choose only prices with positive probability that are in $\{0\} \cup\left[\underline{k}_{2}, \bar{k}_{2}\right]$.

Using these insights about $\pi_{1}$, we can conclude that $\pi_{2}\left(\cdot ; k_{1}\right)$ is piecewise quadratic on $[0,1] \backslash\left[\underline{k}_{2}, \bar{k}_{2}\right]$, where the coefficient in front of the quadratic term is negative and 


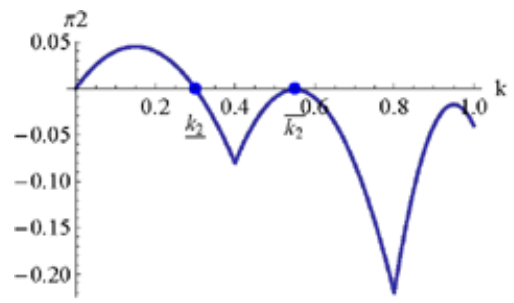

FIGURE 5. Qualitative shapes of buyers' expected profits in period 2.

a multiple of $A / 2-(1-\delta)-\delta A<0$. Hence, all pieces of $\pi_{2}$ are open below. At every cutoff that is chosen with positive probability in period $1, \pi_{2}$ has a kink. Hence, period 2 expected profits are qualitatively as in Figure 5 . Note, however, that $\pi_{2}$ does not have to be piecewise quadratic in $\left[\underline{k}_{2}, \bar{k}_{2}\right]$ as in Figure 5 .

First, note that if $\pi_{1}$ looks like in Figure 4(a), then buyers in period 1 mix between exactly two prices that result in cutoff types 0 and $k_{1}=\underline{k}_{2}=\bar{k}_{2}$. In that case, $\pi_{2}$ has its only kink at $\bar{k}_{2}$, so buyers do not mix between prices in period 2 , but choose a price with probability 1 that results in a cutoff $\bar{k}_{2}$.

If period 2 buyers must mix, then they mix between exactly two cutoffs $\left\{\underline{k}_{2}, \bar{k}_{2}\right\}$. This can be seen as follows: One can infer directly from Lemma 8(ii) that period 2 buyers do not choose prices that result in cutoffs in $\left(\underline{k}_{2}, \bar{k}_{2}\right)$. Moreover, because $\pi_{1}(k ; 0)=0$ on $\{0\} \cup\left[\underline{k}_{2}, \bar{k}_{2}\right]$ only, $\pi_{2}$ can have kinks in that region only. Hence, $\pi_{2}\left(\underline{k}_{2}\right)=\pi_{2}\left(\bar{k}_{2}\right)=0$, $\pi_{2}\left(k ; k_{1}\right) \leq 0$ for $k \geq \underline{k}_{2}$ and the fact that $\pi_{2}$ is piece-wise quadratic on $\left[0, \underline{k}_{2}\right] \cup\left[\bar{k}_{2}, 1\right]$ with parabolas that are open below imply that $\pi_{2}(k)>0$ for $k \in\left(0, \underline{k}_{2}\right)$ and $\pi_{2}\left(k ; k_{1}\right)<0$ for $k \in\left(\bar{k}_{2}, 1\right]$.

Thus, in any equilibrium the support of $K_{1}$ is a subset of $\{0\} \cup\left[\underline{k}_{2}, \bar{k}_{2}\right]$ and the support of $K_{2}$ is $\left\{\underline{k}_{2}, \bar{k}_{2}\right\}$ for some $\underline{k}_{2}, \bar{k}_{2} \in(0,1]$. Let $K_{2}\left(\underline{k}_{2}\right)=q_{2}$ and $K_{1}(0)=q_{1}$, noting that we already know from Lemma 7 that

$$
q_{1}=\frac{\delta-1+\frac{A}{2}}{\delta(1-(1-\alpha) \delta+(1-\alpha) \delta A)} \neq 0 .
$$

STEP 2. In any mixed-strategy equilibrium, there must be non-offers with positive probability in period 1 , i.e., $q_{1}>0$.

Let us assume $q_{1}=0$ and let us denote the smallest element in the support of $K_{1}$ by $\underline{k}<1$. Note that $A k+(1-\delta) B-k(\delta A+(1-\delta))=(1-\delta)(k(A-1)+B) \geq(1-\delta) B(1-k)$, which is strictly positive for $B>0$ and $k<1$. Hence, there exists an $\epsilon>0$ such that $A \underline{k}+(1-\delta) B-(\underline{k}+\epsilon)(\delta A+(1-\delta))>0$. Then $\pi_{2}(\underline{k}+\epsilon)>0$, which is a contradiction to $\underline{k}_{2}<\underline{k}$ being in the support of $K_{2}$.

STEP 3. We have

$$
\underline{k}_{2}=\frac{B(1-(1-\alpha) \delta)}{A(1-\alpha) \delta-(1-\alpha) \delta+1-\frac{A}{2}} \quad \text { and } \quad \bar{k}_{2}=\frac{B\left(1-(1-\alpha) \delta^{2}\right)}{A(1-\alpha) \delta^{2}-(1-\alpha) \delta^{2}+1-\frac{A}{2}} .
$$


In equilibrium, it must hold that $\pi_{2}\left(\underline{k}_{2} ; k_{1}\right)=0$, that is,

$$
\begin{aligned}
\int_{0}^{\underline{k}_{2}} A c+(1- & (1-\alpha) \delta) B-\underline{k}_{2}((1-\alpha) \delta A+1-(1-\alpha) \delta) d c \\
& =\underline{k}_{2} \cdot\left(\frac{A}{2} \underline{k}_{2}+(1-(1-\alpha) \delta) B-\underline{k}_{2}((1-\alpha) \delta A+1-(1-\alpha) \delta)\right)=0
\end{aligned}
$$

which is equivalent to

$$
\underline{k}_{2}=\frac{B(1-(1-\alpha) \delta)}{A(1-\alpha) \delta-(1-\alpha) \delta+1-\frac{A}{2}} .
$$

For $\bar{k}_{2}$, we use that $\pi_{1}\left(\bar{k}_{2} ; 0\right)=0$ since this is equivalent to

$$
\bar{k}_{2} \cdot\left(\frac{A}{2} \bar{k}_{2}+\left(1-(1-\alpha) \delta^{2}\right) B-\delta((1-\alpha) \delta(A-1)+1) \bar{k}_{2}-(1-\delta) \bar{k}_{2}\right)=0
$$

because $K_{2}\left(\bar{k}_{2}\right)=1$. Hence,

$$
\bar{k}_{2}=\frac{B\left(1-(1-\alpha) \delta^{2}\right)}{A(1-\alpha) \delta^{2}-(1-\alpha) \delta^{2}+1-\frac{A}{2}} .
$$

Proof of Lemma 4. Using insights from Proposition 3, we can construct an equilibrium in which buyers in period 1 mix between exactly two cutoffs, which completes the proof of Theorem 2. Here, we calculate the explicit expressions only for $\alpha=0$. The expressions for $\alpha>0$ are much longer, but one can easily check that the construction can be extended to general $\alpha$.

If period 1 buyers mix between exactly two cutoffs 0 and $\bar{k}_{1}$ with $K_{1}(0) \equiv q_{1}$ and $K_{1}\left(\bar{k}_{1}\right)=1-q_{1}$ for some $q_{1} \in(0,1)$. Then the expected profit in period 2 is given by

$$
\pi_{2}\left(k ; k_{1}\right)= \begin{cases}q_{1} \cdot k \cdot\left(\frac{A}{2} k+(1-\delta) B-k(1-\delta+\delta A)\right) & \text { if } k<\bar{k}_{1} \\ \left(q_{1}-1\right) \frac{\bar{k}_{1}}{1-\bar{k}_{1}}\left(\frac{A}{2} \bar{k}_{1}+(1-\delta) B-k(1-\delta+\delta A)\right) & \\ +k\left(\frac{A}{2} k+(1-\delta) B-k(1-\delta+\delta A)\right) & \text { if } k>\bar{k}_{1} .\end{cases}
$$

Note that both parts are quadratic in $k$ and that $\pi_{2}$ is continuous everywhere. Moreover, in both parts the coefficient in front of $k^{2}$ is negative. The first part is equal to zero if

$$
k \in\left\{0, \frac{B(1-\delta)}{1-\delta+\delta A-\frac{A}{2}}\right\}
$$

and the second part must only have one zero in equilibrium, i.e., it must hold that the discriminant is zero,

$$
\begin{aligned}
& {\left[(1-\delta) B\left(q_{1}+\frac{1}{1-\bar{k}_{1}}\left(1-q_{1}\right)\right)-\left(q_{1}-1\right) \frac{\bar{k}_{1}}{1-\bar{k}_{1}}(1-\delta+\delta A)\right]^{2}} \\
& \quad=(2 A-4+4 \delta(1-A))\left(q_{1}+\frac{1}{1-\bar{k}_{1}}\left(1-q_{1}\right)\right)\left(q_{1}-1\right) \frac{\bar{k}_{1}}{1-\bar{k}_{1}}\left(\frac{A}{2} \bar{k}_{1}+(1-\delta) B\right),
\end{aligned}
$$


and the null must be at

$$
\bar{k}_{2}=-\frac{(1-\delta) B\left(r+\frac{1}{1-\bar{k}_{1}}\left(1-q_{1}\right)\right)-\left(q_{1}-1\right) \frac{\bar{k}_{1}}{1-\bar{k}_{1}}(1-\delta+\delta A)}{(A-2+2 \delta(1-A))\left(q_{1}+\frac{1}{1-\bar{k}_{1}}\left(1-q_{1}\right)\right)} .
$$

We can solve these two equations for $\bar{k}_{1}, q_{1}$. For $\alpha=0$ this yields

$$
\bar{k}_{1}=\frac{2 B(1-\delta)\left(-2 \delta^{3}+2 \delta^{3} A+4 \delta^{2} A-2 \delta^{2}+2 \delta-A+2\right)}{\left(-2 \delta^{2}+2 \delta^{2} A+2 \delta A-A+2\right)\left(-2 \delta^{2}+2 \delta^{2} A+2-A\right)}
$$

such that $0 \leq \underline{k}_{2}<\bar{k}_{1}<\bar{k}_{2} \leq 1$.

Figure 1 illustrates for $v(c)=(1+c) / 2$ the expected profit functions $\pi_{1}$ and $\pi_{2}$ in the equilibrium. It highlights how cutoffs in period 1 must correspond to kinks of $\pi_{2}$ and how cutoffs in the second period must correspond to kinks of $\pi_{1}$. Other equilibria, in which period 1 buyers 1 mix between $\{0\}$ and several prices in $\left(\underline{k}_{2}, \bar{k}_{2}\right)$, can coexist.

Proof of Lemma 5. We first show that the expected cutoffs in period 1 are constant across all mixed-strategy equilibria with private offers and are equal to

$$
\int_{0}^{\bar{k}_{2}} \tilde{k} d K_{1}(\tilde{k})=\frac{\left(1-\bar{k}_{2}\right)\left(1-\frac{\delta}{1+\delta} \bar{k}_{2}\right)}{1-\bar{k}_{2} \cdot \frac{(1+\delta)(1-\delta+A \delta)-A / 2}{(1+\delta)(1-\delta+A \delta)}}+\frac{1+2 \delta}{1+\delta} \bar{k}_{2}-1 .
$$

Moreover,

$$
\int_{0}^{\bar{k}_{2}} \frac{1}{1-\tilde{k}} d K_{1}(\tilde{k})=\frac{1}{1-\bar{k}_{2} \cdot \frac{(1+\delta)(1-\delta+A \delta)-A / 2}{(1+\delta)(1-\delta+A \delta)}}
$$

must hold. To prove this, note that in any equilibrium it must hold that $\pi_{2}\left(\bar{k}_{2} ; K_{1}\right)=0$ and for all $d>\bar{k}_{2}, \pi_{2}\left(k ; K_{1}\right) \leq 0$, i.e.,

$$
\begin{aligned}
& \int_{0}^{\bar{k}_{2}} \int_{0}^{c} \frac{1}{1-\tilde{k}} d K_{1}(\tilde{k})\left(A c+B-\left((1-\delta+A \delta) \bar{k}_{2}+\delta B\right)\right) d c=0 \\
& \int_{0}^{d} \int_{0}^{c} \frac{1}{1-\tilde{k}} d K_{1}(\tilde{k})(A c+B-((1-\delta+A \delta) d+\delta B)) d c \leq 0 \quad \forall d>\bar{k}_{2} .
\end{aligned}
$$

Let us first simplify the first equality. By applying Fubini's theorem and then noting that $\left(\bar{k}_{2}-k\right) /(1-k)=1+\left(\bar{k}_{2}-1\right) /(1-k)$ and $\left(\bar{k}_{2}^{2}-k^{2}\right) /(1-k)=1+k+\left(\bar{k}_{2}^{2}-1\right) /(1-k)$, we can deduce

$$
\begin{aligned}
\int_{0}^{\bar{k}_{2}} & \int_{0}^{c} \frac{1}{1-\tilde{k}} d K_{1}(\tilde{k})\left(A c+B-\left((1-\delta+A \delta) \bar{k}_{2}+\delta B\right)\right) d c \\
\quad= & \frac{A}{2} \int_{0}^{\bar{k}_{2}} \frac{\bar{k}_{2}^{2}-\tilde{k}^{2}}{1-\tilde{k}} d K_{1}(\tilde{k})+\left((1-\delta) B-(1-\delta+A \delta) \bar{k}_{2}\right) \int_{0}^{\bar{k}_{2}} \frac{\bar{k}_{2}-\tilde{k}}{1-\tilde{k}} d K_{1}(\tilde{k}) \\
\quad= & \frac{A}{2}+(1-\delta) B-(1-\delta+A \delta) \bar{k}_{2}+\frac{A}{2} \int_{0}^{\bar{k}_{2}} \tilde{k} d K_{1}(\tilde{k})
\end{aligned}
$$




$$
\begin{array}{r}
+\int_{0}^{\bar{k}_{2}} \frac{1}{1-\tilde{k}} d K_{1}(\tilde{k})\left(\left(\bar{k}_{2}^{2}-1\right) \frac{A}{2}+\left(\bar{k}_{2}-1\right)\left((1-\delta) B-(1-\delta+A \delta) \bar{k}_{2}\right)\right) \\
=\frac{A}{2} \cdot\left(1-\frac{1+2 \delta}{1+\delta} \bar{k}_{2}+\int_{0}^{\bar{k}_{2}} \tilde{k} d K_{1}(\tilde{k})+\left(\bar{k}_{2}-1\right)\left(1-\frac{\delta}{1+\delta} \bar{k}_{2}\right) \int_{0}^{\bar{k}_{2}} \frac{1}{1-\tilde{k}} d K_{1}(\tilde{k})\right) .
\end{array}
$$

Thus, in equilibrium,

$$
1-\frac{1+2 \delta}{1+\delta} \bar{k}_{2}+\int_{0}^{\bar{k}_{2}} \tilde{k} d K_{1}(\tilde{k})=\left(1-\bar{k}_{2}\right)\left(1-\frac{\delta}{1+\delta} \bar{k}_{2}\right) \int_{0}^{\bar{k}_{2}} \frac{1}{1-\tilde{k}} d K_{1}(\tilde{k})
$$

must hold. To simplify the second inequality, we can use that $\pi_{2}\left(\bar{k}_{2} ; k_{1}\right)=0$, and see that for $d>\bar{k}_{2}$,

$$
\begin{aligned}
& \int_{0}^{d} \int_{0}^{c} \frac{1}{1-\tilde{k}} d K_{1}(\tilde{k})(A c+B(1-\delta)-(1-\delta+A \delta) d) d c \\
& =\int_{0}^{\bar{k}_{2}} \int_{0}^{c} \frac{1}{1-\tilde{k}} d K_{1}(\tilde{k}) d c\left(\bar{k}_{2}-d\right)(1-\delta+A \delta) \\
& \quad+\int_{\bar{k}_{2}}^{d} \int_{0}^{\bar{k}_{2}} \frac{1}{1-\tilde{k}} d K_{1}(\tilde{k})(A c+B(1-\delta)-(1-\delta+A \delta) d) d c \\
& =\left(d-\bar{k}_{2}\right)\left[\int_{0}^{\bar{k}_{2}} \frac{1}{1-\tilde{k}} d K_{1}(\tilde{k})(\frac{A}{2} \bar{k}_{2}+B(1-\delta)-\underbrace{\left(1-\delta+A \delta-\frac{A}{2}\right)}_{>0} d)\right. \\
& \left.\quad-\int_{0}^{\bar{k}_{2}} \int_{0}^{c} \frac{1}{1-\tilde{k}} d K_{1}(\tilde{k}) d c(1-\delta+A \delta)\right]
\end{aligned}
$$

is quadratic in $d$ and the parabola is open below. The parabola has a zero at $\bar{k}_{2}$ and we will show in the following text that it cannot have another zero. If $\pi_{2}\left(k^{\prime} ; k_{1}\right)=0$ for a $k^{\prime}>\bar{k}_{2}$, then $\pi_{2}$ is positive on $\left(\bar{k}_{2}, k^{\prime}\right)$, which cannot hold in equilibrium. If the parabola (if it was extended to values smaller than $\bar{k}_{2}$ ) has a zero at a $k^{\prime}<\bar{k}_{2}$ and if the support of $K_{1}$ does not contain $\left(\bar{k}_{2}-\epsilon, \bar{k}_{2}\right)$ for an $\epsilon>0$, then $\pi_{2}\left(k ; k_{1}\right)>0$ for $k \in\left(\bar{k}_{2}-\epsilon, \bar{k}_{2}\right)$, which leads to a contradiction. Finally, if there is continuous mixing on some $\left(\bar{k}_{2}-\epsilon, \bar{k}_{2}\right)$, then since the slope from the right of $\pi_{2}$ is negative at $\pi_{2}$, the slope from the left must also be negative because

$$
\begin{aligned}
\frac{\partial}{\partial k_{2}} \pi_{2}\left(k_{2}\right)= & \frac{\partial}{\partial k_{2}} \int_{0}^{k_{2}} \int_{0}^{c} \frac{1}{1-\tilde{k}} d K_{1}(\tilde{k})\left(A c-(1-\delta+A \delta) k_{2}+B(1-\delta)\right) d c \\
=\int_{0}^{k_{2}} \frac{1}{1-\tilde{k}} d K_{1}(\tilde{k})(1-\delta)\left(A k_{2}-k_{2}+B\right) & -(1-\delta+A \delta) \int_{0}^{k_{2}} \int_{0}^{c} \frac{1}{1-\tilde{k}} d K_{1}(\tilde{k}) d c
\end{aligned}
$$

and $k_{2}(A-1)+B>0$. This again cannot hold in equilibrium. As a result, the parabola can only have one zero $\bar{k}_{2}$ and it follows from plugging in the value of $\bar{k}_{2}$ calculated in 
Proposition 3 that

$$
\begin{aligned}
& \frac{\int_{0}^{\bar{k}_{2}} \frac{1}{1-\tilde{k}} d K_{1}(\tilde{k})\left(\frac{A}{2} \bar{k}_{2}+B(1-\delta)\right)-\int_{0}^{\bar{k}_{2}} \int_{0}^{c} \frac{1}{1-\tilde{k}} d K_{1}(\tilde{k}) d c(1-\delta+A \delta)}{\int_{0}^{\bar{k}_{2}} \frac{1}{1-\tilde{k}} d K_{1}(\tilde{k})\left(1-\delta+A \delta-\frac{A}{2}\right)}=\bar{k}_{2} \\
& \Leftrightarrow \frac{A}{2} \bar{k}_{2}+B(1-\delta)-\frac{\int_{0}^{\bar{k}_{2}} \int_{0}^{c} \frac{1}{1-\tilde{k}} d K_{1}(\tilde{k}) d c(1-\delta+A \delta)}{\int_{0}^{\bar{k}_{2}} \frac{1}{1-\tilde{k}} d K_{1}(\tilde{k})} \\
& =\frac{B\left(1-\delta^{2}\right)\left(1-\delta-\frac{A}{2}+A \delta\right)}{\left(1-\delta^{2}-\frac{A}{2}+A \delta^{2}\right)} \\
& \Leftrightarrow \quad(1-\delta) B \cdot \frac{\frac{A}{2}}{\left(1-\delta^{2}-\frac{A}{2}+A \delta^{2}\right)(1-\delta+A \delta)}=\frac{\int_{0}^{\bar{k}_{2}} \int_{0}^{c} \frac{1}{1-\tilde{k}} d K_{1}(\tilde{k}) d c}{\int_{0}^{\bar{k}_{2}} \frac{1}{1-\tilde{k}} d K_{1}(\tilde{k})} \\
& \Leftrightarrow \quad \bar{k}_{2} \cdot \frac{\frac{A}{2}}{1-\delta^{2}+A \delta+A \delta^{2}}=\frac{1+\left(\bar{k}_{2}-1\right) \int_{0}^{\bar{k}_{2}} \frac{1}{1-\tilde{k}} d K_{1}(\tilde{k})}{\int_{0}^{\bar{k}_{2}} \frac{1}{1-\tilde{k}} d K_{1}(\tilde{k})} \\
& \Leftrightarrow \quad \int_{0}^{\bar{k}_{2}} \frac{1}{1-\tilde{k}} d K_{1}(\tilde{k})=\frac{1}{\frac{(A / 2) \bar{k}_{2}}{1-\delta^{2}+A \delta+A \delta^{2}}-\bar{k}_{2}+1} \text {. }
\end{aligned}
$$

This proves (10). Plugging (10) into (11) shows (9).

We can now easily calculate the difference between the expected period 1 cutoff with private offers and the period 1 cutoff with public offer using (6), (8), Proposition 5, and Proposition 3, and we see that it is positive:

$$
\begin{aligned}
\int_{0}^{\bar{k}_{2}} \tilde{k} d K_{1}(\tilde{k})-k_{1}^{*} & \left(1-\frac{B\left(1-\delta^{2}\right)}{A \delta^{2}-\delta^{2}+1-A / 2}\right)\left(1-\frac{\delta}{1+\delta} \frac{B\left(1-\delta^{2}\right)}{A \delta^{2}-\delta^{2}+1-A / 2}\right) \\
1-\bar{k}_{2} \cdot \frac{(1+\delta)(1-\delta+A \delta)-A / 2}{(1+\delta)(1-\delta+A \delta)} & +\frac{1+2 \delta}{1+\delta} \frac{B\left(1-\delta^{2}\right)}{A \delta^{2}-\delta^{2}+1-\frac{A}{2}}-1 \\
= & \frac{(1-A)(1-\delta)\left(-4+4 A-A^{2}+8 \delta-6 A \delta+2 A^{2} \delta-4 \delta^{2}+2 A \delta^{2}+2 A^{2} \delta^{2}\right)}{\left(2-A-2 \delta^{2}+2 A \delta^{2}\right)\left(4-4 A+A^{2}-8 \delta+10 A \delta-2 A^{2} \delta+4 \delta^{2}-6 A \delta^{2}+2 A^{2} \delta^{2}\right)} \\
= & \frac{1}{2} \bar{k}_{\Delta} \cdot \frac{2 A(1-\delta)-(A-1)^{2}(1-\delta)^{2}-3(1-\delta)^{2}+\delta^{2} A}{\left(4-4 A+A^{2}\right)\left(1-2 \delta+\delta^{2}\right)+2 A \delta-2 A \delta^{2}+A^{2} \delta^{2}} \\
\geq & \frac{1}{2} \bar{k}_{\Delta} \cdot \frac{(1-\delta)^{2}-(A-1)^{2}(1-\delta)^{2}+\delta^{2} A}{\left(4-4 A+A^{2}\right)\left(1-2 \delta+\delta^{2}\right)+2 A \delta-2 A \delta^{2}+A^{2} \delta^{2}}>0
\end{aligned}
$$

for $\delta>1-A / 2$ and $A+B=1$. 


\section{Proofs: Robustness and generalizations}

Proof of Theorem 4(i). We show that if all buyers choose pricing strategies that result in a cutoff seller $\kappa_{t}\left(k_{t-\Delta}\right)$ (defined below) given they believe the current cutoff is $k_{t-\Delta}$, this constitutes an equilibrium. To this end, define $\kappa_{t}(\cdot)$ inductively for $t=0, \Delta, \ldots, 1-\Delta$ as follows. First, using $p_{1-\Delta}(k)=(1-\alpha) \delta v(k)+(1-(1-\alpha) \delta) k$, it follows that

$$
\kappa_{1-\Delta}\left(k_{1-2 \Delta}\right)=\sup \left\{k \in\left[k_{1-2 \Delta}, 1\right] \mid \frac{1}{1-F\left(k_{1-2 \Delta}\right)} \int_{k_{1-2 \Delta}}^{k}\left(v(c)-p_{1-\Delta}(k)\right) f(c) d c>0\right\}
$$

is left-continuous (we define $\kappa_{1-\Delta}\left(k_{1-2 \Delta}\right)=k_{1-2 \Delta}$ if the set over which we compute the sup is empty). Then

$$
p_{1-2 \Delta}(k)=\delta p_{1-\Delta}\left(c_{1-\Delta}\left(k_{1-2 \Delta}\right)\right)+(1-\delta) k
$$

is left-continuous.

Next, we show that given left-continuous $\kappa_{t+\Delta}(k)$, it follows that for $t<1-\Delta$ and $p_{t}(k)=\delta p_{t+\Delta}\left(\kappa_{t+\Delta}(k)\right)+(1-\delta) k$,

$$
\kappa_{t}\left(k_{t-\Delta}\right)=\sup \left\{k \in\left[k_{T-2 \Delta}, 1\right] \mid \frac{1}{1-F\left(k_{T-2 \Delta}\right)} \int_{k_{T-2 \Delta}}^{k}\left(v(c)-p_{t}(k)\right) f(c) d c>0\right\}
$$

(with $\sup \varnothing=k_{t-\Delta}$ ) is left-continuous.

STEP 1. If $\pi_{t}\left(k ; k_{t-\Delta}\right)$ is left-continuous in $k$, then $\kappa_{t}$ is increasing.

Because of left continuity of $\pi_{t}\left(\cdot ; k_{t-\Delta}\right)$, we either have $\pi_{t}\left(\kappa_{t}\left(k_{t-\Delta}\right), k_{t-\Delta}\right)>0$ or $\pi_{t}\left(\kappa_{t}\left(k_{t-\Delta}\right), k_{t-\Delta}\right)=0$. Moreover, note that $\pi_{t}\left(k ; k_{t-\Delta}\right)$ is always differentiable in $k_{t-\Delta}$. Let us consider an arbitrary $k_{t-\Delta}$ and an infinitesimal increase in $k_{t-\Delta}$. If $\pi_{t}\left(\kappa_{t}\left(k_{t-\Delta}\right), k_{t-\Delta}\right)>0$, there exists an $\epsilon>0$ so that $\pi_{t}\left(\kappa_{t}\left(k_{t-\Delta}\right), k_{t-\Delta}+\gamma\right)>0$ for all $\gamma<\epsilon$. Hence, $\kappa_{t}\left(k_{t-\Delta}+\gamma\right)>\kappa_{t}\left(k_{t-\Delta}\right)$ for all $\gamma<\epsilon$. Furthermore, if $\pi_{t}\left(\kappa_{t}\left(k_{t-\Delta}\right), k_{t-\Delta}\right)=$ 0 , then

$$
\begin{aligned}
& \left.\frac{\partial}{\partial k_{t-\Delta}} \pi_{t}\left(k ; k_{t-\Delta}\right)\right|_{k=\kappa_{t}\left(k_{t-\Delta}\right)} \\
& =\frac{f\left(k_{t-\Delta}\right)}{1-F\left(k_{t-\Delta}\right)} \\
& \quad \cdot\left[\frac{1}{1-F\left(k_{t-\Delta}\right)} \int_{k_{t-\Delta}}^{\kappa_{t}\left(k_{t-\Delta}\right)}\left(v(c)-p_{t}\left(\kappa_{t}\left(k_{t-\Delta}\right)\right)\right) f(c) d c-\left(v\left(k_{t-\Delta}\right)-p_{t}\left(c_{t}^{*}\left(k_{t-\Delta}\right)\right)\right)\right] \\
& =-\frac{f\left(k_{t-\Delta}\right)}{1-F\left(k_{t-\Delta}\right)}\left(v\left(k_{t-\Delta}\right)-p_{t}\left(\kappa_{t}\left(k_{t-\Delta}\right)\right)\right)>0 .
\end{aligned}
$$

This is the case because if we had $v\left(k_{t-\Delta}\right)-p_{t}\left(\kappa_{t}\left(k_{t-\Delta}\right)\right) \geq 0$, then

$$
\int_{k_{t-\Delta}}^{\kappa_{t}\left(k_{t-\Delta}\right)}\left(v(c)-p_{t}\left(\kappa_{t}\left(k_{t-\Delta}\right)\right)\right) f(c) d c>0
$$


$v(\cdot)$ being increasing. This is a contradiction to the zero-profit assumption $\pi_{t}\left(\kappa_{t}\left(k_{t-\Delta}\right)\right.$, $\left.k_{t-\Delta}\right)=0$. Hence, $\kappa_{t}(\cdot)$ is increasing at $k_{t-\Delta}$.

STEP 2. The terms $\kappa_{t}(\cdot), p_{t}(\cdot)$, and $\pi_{t}\left(\cdot ; k_{t-\Delta}\right)$ are left-continuous.

We argue by backward induction in $t$. The term $p_{1-\Delta}(\cdot)$ is left-continuous because $v$ is continuous and, hence, $\pi_{1-\Delta}\left(k_{1-2 \Delta} ; k\right)$ is left-continuous in $k$. (It is even continuous.) Let $k_{1-2 \Delta}^{(n)} \uparrow k_{1-2 \Delta}$. Then $\kappa_{1-\Delta}^{*}\left(k_{1-2 \Delta}^{(n)}\right) \leq \kappa_{1-\Delta}\left(k_{1-2 \Delta}\right)$ for all $n$ and $\kappa_{1-\Delta}\left(k_{1-2 \Delta}^{(n)}\right)$ is an increasing sequence by Step 1 . Hence, $\lim _{n \rightarrow \infty} \kappa_{1-\Delta}^{*}\left(k_{1-2 \Delta}^{(n)}\right)$ exists. We will show next that $\lim _{n \rightarrow \infty} \kappa_{1-\Delta}\left(k_{1-2 \Delta}^{(n)}\right)=\kappa_{1-\Delta}\left(k_{1-2 \Delta}\right)$. Therefore, consider an arbitrary sequence $k^{(m)} \uparrow$ $\kappa_{1-\Delta}\left(k_{1-2 \Delta}\right)$ such that $\pi_{1-\Delta}\left(k^{(m)} ; k_{1-2 \Delta}\right)>0$ (which must exist by definition of $\kappa_{1-\Delta}$ ). Then, for any $m$, there exists an $n(m)$ such that $\pi_{1-\Delta}\left(k^{(m)} ; k_{1-2 \Delta}^{(n)}\right)>0$ for all $n \geq n(m)$ because $\pi_{1-\Delta}(k ; \cdot)$ is continuous for all $k$. Hence, $k^{(m)} \leq \kappa_{1-\Delta}\left(k_{1-2 \Delta}^{(n(m))}\right) \leq \kappa_{1-\Delta}\left(k_{1-2 \Delta}\right)=$ $\lim _{m \rightarrow \infty} k^{(m)}$. Hence, $\lim _{n \rightarrow \infty} \kappa_{1-\Delta}\left(k_{1-2 \Delta}^{(n)}\right)=\lim _{m \rightarrow \infty} \kappa_{1-\Delta}\left(k_{1-2 \Delta}^{(n(m))}\right)=\kappa_{1-\Delta}\left(k_{1-2 \Delta}\right)$ and, thus, $\kappa_{1-\Delta}(\cdot), p_{1-2 \Delta}(\cdot)$ and $\pi_{1-2 \Delta}\left(\cdot ; k_{1-3 \Delta}\right)$ are left-continuous.

Let us now assume that $c_{t+\Delta}(\cdot), p_{t}(\cdot)$ and $\pi_{t}\left(\cdot ; k_{t-\Delta}\right)$ are left-continuous. Hence, $\kappa_{t}(\cdot)$ is increasing by Step 1 . The rest of the argument works analogously to the preceding text, so that $\kappa_{t}(\cdot), p_{t-\Delta}(\cdot)$ and $\pi_{t-\Delta}\left(\cdot ; k_{t-2 \Delta}\right)$ are left-continuous for all $t$.

Hence, buyers do not make negative expected profits because

$$
k \mapsto \frac{1}{1-F\left(k_{t-\Delta}\right)} \int_{k_{t-\Delta}}^{k}\left(v(c)-\left(\delta p_{t+1}\left(\kappa_{t+\Delta}(k)\right)+(1-\delta) k\right)\right) f(c) d c
$$

is left-continuous. The equilibrium cutoffs $\left(k_{0}^{*}, \ldots, k_{1-\Delta}^{*}\right)$ are then, given by $k_{0}^{*}=\kappa_{0}(0)$, $\ldots, k_{1-\Delta}=\kappa_{1-\Delta}\left(\kappa_{1-2 \Delta}\left(\ldots \kappa_{0}(0)\right)\right)$. None of the buyers has an incentive to deviate from this equilibrium, since by increasing the price offer, buyers will either make zero or negative expected profits by definition of $\kappa_{t}(\cdot)$, and by decreasing the price, they will not receive the good and make zero expected profits. Note that for some $v(c)$ and $F_{1}$ there could be multiple equilibria because there can be several prices that result in zero expected profits for the buyers.

Parts (ii) and (iii) are proven in the main part of the paper.

Proof of Proposition 4. Let $B=0$ and $A<2$. The zero-profit condition implies that the cutoff at time 0 must satisfy

$$
p_{0}=v\left(\frac{k_{0}}{2}\right)=\frac{A}{2} k_{0}
$$

Moreover, it must hold that

$$
p_{0} \geq(1-\delta) k_{0}+\delta p_{1} \geq k_{0}
$$

However, this can never hold simultaneously for $A<2$ except if $k_{0}=0$. Hence, in the unique pure-strategy equilibrium (with private and public offers), there is no trade before the deadline. 


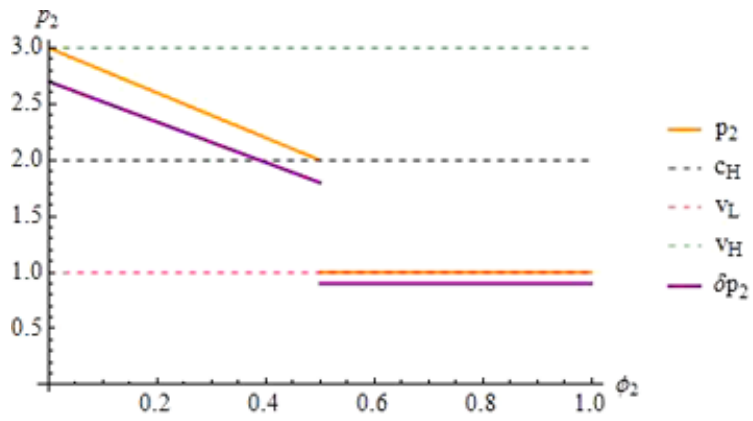

Figure 6. Period 2 price.

Proof of Proposition 5. Consider a situation with $v_{H} \geq c_{H}$ and $v_{L}>c_{L}=0$, where the seller's valuation is $v_{L}$ with probability $\phi$. The static lemon's condition (LC) is satisfied if

$$
\phi v_{L}+(1-\phi) v_{H}<c_{H}
$$

Finally, denote the fraction of $v_{L}$ sellers such that the lemons condition is just satisfied by $\phi^{*}$, i.e.,

$$
\phi^{*} v_{L}+\left(1-\phi^{*}\right) v_{H}=c_{H} .
$$

We solve the game by backward induction. Given the belief $\phi_{2}$ about the fraction of $v_{L}$ sellers in the market, buyers' expected period 2 profits are given by

$$
\pi_{2}(p)= \begin{cases}\phi_{2} v_{L}+\left(1-\phi_{2}\right) v_{H}-p & \text { if } p \geq c_{H} \\ v_{L}-p & \text { if } c_{L}<p<c_{H} \\ 0 & \text { otherwise }\end{cases}
$$

if they sell at a price $p$. Since buyers compete in a Bertrand fashion, the equilibrium price is

$$
p_{2}^{*}\left(\phi_{2}\right)= \begin{cases}\phi_{2} v_{L}+\left(1-\phi_{2}\right) v_{H} & \text { if } \phi_{2}<\phi^{*} \\ \left\{\phi_{2} v_{L}+\left(1-\phi_{2}\right) v_{H}, v_{L}\right\} & \text { if } \phi_{2}=\phi^{*} \\ v_{L} & \text { if } \phi_{2}>\phi^{*}\end{cases}
$$

If the LC is satisfied with $\phi_{2}$, only low types trade and $p_{2}^{*}=v_{L}$.

The price in the continuation equilibrium is as in Figure 6. If the LC is satisfied, then the period 1 price is always $p_{1}=v_{L}$. Moreover, the following statements hold:

(i) If $\delta \leq v_{L} / v_{H}$, then all $v_{L}$ sellers trade in period 1 and $p_{2}=v_{H}$.

(ii) If $v_{L} / v_{H}<\delta<v_{L} / c_{H}$, then in period 1 enough $v_{L}$ sellers trade such that in period 2,

$$
\phi_{2} v_{L}+\left(1-\phi_{2}\right) v_{H}=\frac{v_{L}}{\delta} .
$$

Note that $\phi_{2}<\phi$, such that in period 2, $p_{2}=\phi_{2} v_{L}+\left(1-\phi_{2}\right) v_{H}$. 
(iii) If $\frac{v_{L}}{c_{H}}<\delta$, then in period 1 enough $v_{L}$ sellers trade such that in period 2, $\phi_{2}=$ $\phi^{*}$, such that in period 2, buyers are indifferent between bidding $c_{H}=\phi^{*} v_{L}+$ $\left(1-\phi^{*}\right) v_{H}$ and $v_{L}$. They mix between the two such that

$$
\mathbb{E}\left[p_{2}\right]=\frac{v_{L}}{\delta} .
$$

These are by construction all equilibria with both private and public offers.

\section{The role of distress with many periods}

The role of distress $(\alpha>0)$ can also be generalized to a setup with more than two trading opportunities with linear valuations and uniformly distributed costs. In particular, we can show that even as $\Delta \rightarrow 0$, there must be a positive mass of trade at the deadline. This is formalized in the following lemma.

Lemma 9. For any $\alpha>0$, with public offers, as $\Delta \rightarrow 0$, trade at time $1-\Delta$ is strictly bounded away from zero.

Proof. At time $1-\Delta$, prices are given by

$$
p_{1-\Delta}(k)=(1-(1-\alpha) \delta) \underbrace{k}_{<v(k)}+(1-\alpha) \delta v(k) .
$$

Thus, there must be positive trade at time $1-\Delta$ because

$$
\pi_{1-\Delta}\left(k_{1-2 \Delta}+\epsilon ; k_{1-2 \Delta}\right)=\int_{k_{1-2 \Delta}}^{k_{1-2 \Delta}+\epsilon}\left(v(c)-p_{1-\Delta}\left(k_{1-2 \Delta}+\epsilon\right)\right) f(c) d c
$$

and, for small $\epsilon$,

$$
\lim _{\Delta \rightarrow 0} v\left(k_{1-2 \Delta}\right)-p_{1-\Delta}\left(k_{1-2 \Delta}+\epsilon\right)>0 .
$$

Consequently, trade in period $1-\Delta$ is bounded away from zero as $\Delta \rightarrow 0$. This shows that with public offers, as $\Delta \rightarrow 0$, trade at time $1-\Delta$ is strictly bounded away from zero.

Proposition 6 (Quiet periods). With public offers, for any $\alpha>0$ there exists a $\delta^{* *}<1$ such that if $\delta>\delta^{* *}$, there will be no trade in at least one period preceding the deadline and possibly no period but the last period.

Proof. By Lemma 9, $p_{1-\Delta}\left(k_{1-\Delta}\right)$ is greater and bounded away from $v\left(k_{1-2 \Delta}\right)$. If there was trade in period $1-2 \Delta$, then the highest type trading in that period $k_{1-2 \Delta}$ can at most get a price $v\left(k_{1-2 \Delta}\right)$. Alternatively, the period after, he can buy at a price $p_{1-\Delta}\left(k_{1-\Delta}\right)$ that is strictly greater than and bounded away (for all $\Delta$ ) from $v\left(k_{1-2 \Delta}\right)$. Hence, for small enough $\Delta$, there cannot be trade in period $1-2 \Delta$. In other words, for large $\delta$, there must be a quiet period before the deadline. 


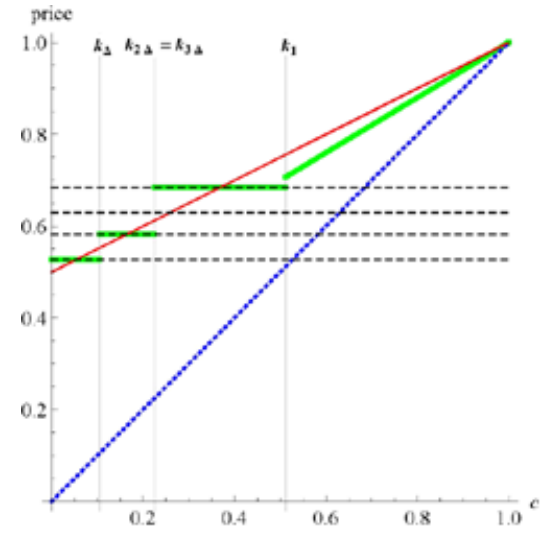

(a) Prices as a function of seller types for $\Delta=\frac{1}{4}$

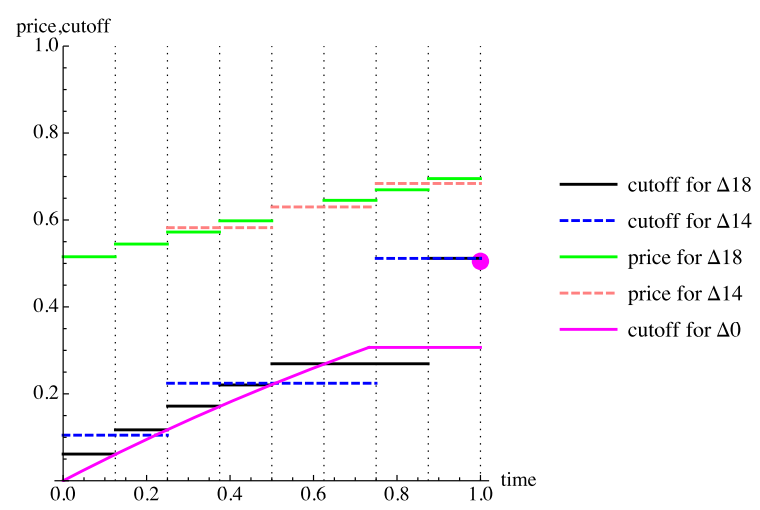

(b) Prices and cutoffs over time for $\Delta \in\left\{\frac{1}{4}, \frac{1}{8}\right\}$

Figure 7. Pure-strategy equilibria with $r=0.5, v(c)=(c+1) / 2, \alpha=0.2$.

By the same logic, for a given fixed $N$, when $\delta^{N}$ is large, there will be $N$ quiet periods before the deadline.

Note that this is in stark contrast to the no trade result in HV. Recall that they have trade only in the first period with public offers while potentially we have no trade but in the last period. The differences in outcomes are caused by two differences in the models: first, we have intra- and inter-period competition while HV have only the latter; second, we have a short horizon with a destruction of continuation surplus at the deadline.

In Figure 7 we plot the unique pure-strategy equilibria with public offers using $v(c)=$ $(c+1) / 2$ for $\Delta \in\left\{0, \frac{1}{4}, \frac{1}{8}\right\}, r=0.5$, and $\alpha=0.2 .^{20}$ Indeed, in the limit as $\Delta \rightarrow 0$, with public offers, there is a mass of trade at time 1 and some "quiet periods" in which no trade takes place. In particular, in the last period it must hold that

$$
p_{1}=\alpha k_{1}+(1-\alpha) v\left(k_{1}\right)=\mathbb{E}\left[v(c) \mid c \in\left[k_{1-}, k_{1}\right]\right],
$$

where at time 1 the mass of seller types $\left[k_{1-}, k_{1}\right]$ trades (where $k_{1-}$ is the limiting cutoff as time approaches 1 from the left). Moreover, before the quiet period, there must be continuous trading over time. In particular, for $A=B=0.5$ the cutoff is given by

$$
k_{t}=1-e^{-r t} .
$$

Finally, the condition that seller $k_{1-}$ must be indifferent between buying just before the quiet period starts and waiting until time 1 pins down the evolution of cutoffs over time. It turns out that the quiet period before deadline is caused not only by distress, but also by the market structure. In particular, we can contrast equilibrium dynamics in our model with intra- and inter-period competition to a monopoly case. As shown in Fuchs and Skrzypacz (2013a), in a model with one long-lived buyer, the distress at deadline also induces an atom of trade at the end, but the quiet period does not arise there.

\footnotetext{
${ }^{20}$ See Figure 3 for a comparison when $\alpha=0$.
} 


\section{REFERENCES}

Akerlof, George A. (1970), “The market for 'lemons': Quality uncertainty and the market mechanism.” The Quarterly Journal of Economics, 84, 488-500. [1104]

Asriyan, Vladimir, William Fuchs, and Brett Green (2015), "Information spillovers in asset markets with correlated values.” Working paper. [1107, 1127]

Bergemann, Dirk and Johannes Hörner (2014), "Should auctions be transparent?" Cowles discussion paper, Yale University. [1107]

Boehmer, Ekkehart, Gideon Saar, and Lei Yu (2005), "Lifting the veil: An analysis of pretrade transparency at the NYSE.” Journal of Financial Economics, 60, 783-815. [1108]

Buti, Sabrina and Barbara Rindi (2013), "Undisclosed orders and optimal submission strategies in a dynamic limit order market." Journal of Financial Economics, 109, 797812. [1108]

Camargo, Braz and Benjamin Lester (2014), “Trading dynamics in decentralized markets with adverse selection.” Journal of Economic Theory, 153, 534-568. [1126]

Daley, Brendan and Brett Green (2012), "Waiting for news in the market for lemons." Econometrica, 80, 1433-1504. [1108, 1126]

Deneckere, Raymond and Meng-Yu Liang (2006), "Bargaining with interdependent values.” Econometrica, 74, 1309-1364. [1107, 1127]

Diamond, Peter A. (1971), “A model of price adjustment.” Journal of Economic Theory, 3, 156-168. [1106]

Flood, Mark D., Ronald Huisman, Kees G. Koedijk, and Ronald J. Mahieu (1999), "Quote disclosure and price discovery in multiple-dealer financial markets." Review of Financial Studies, 12, 37-59. [1108]

Fuchs, William and Andrzej Skrzypacz (2013a), "Bargaining with deadlines and private information.” American Economic Journal: Microeconomics, 5, 219-243. [1142]

Fuchs, William and Andrzej Skrzypacz (2013b), "Bridging the gap: Bargaining with interdependent values.” Journal of Economic Theory, 148, 1226-1236. [1108]

Fuchs, William and Andrzej Skrzypacz (2015), "Government interventions in a dynamic market with adverse selection.” Journal of Economic Theory, 158, 371-406. [1106, 1108]

Hayek, Friedrich August (1945), "The use of knowledge in society.” American Economic Review, 35, 519-530. [1127]

Hörner, Johannes and Nicolas Vieille (2009), "Public vs. private offers in the market for lemons." Econometrica, 77, 29-69. [1106]

Janssen, Maarten C. W. and Santanu Roy (2002), "Dynamic trading in a durable good market with asymmetric information.” International Economic Review, 43, 257-282. [1108] 
Kaya, Ayca and Quingmin Liu (2015), “Transparency and price formation.” Theoretical Economics, 10, 341-383. [1105, 1107]

Kim, Kyungmin (2015), “Information about sellers' past behavior in the market for lemons.” Unpublished manuscript, University of Iowa. [1107]

Kremer, Ilan and Andrzej Skrzypacz (2007), "Dynamic signaling and market breakdown.” Journal of Economic Theory, 133, 58-82. [1107]

Madhavan, Ananth, David Porter, and Daniel Weaver (2005), “Should securities markets be transparent?” Journal of Financial Markets, 8, 265-287. [1108]

Nöldeke, Georg and Eric Van Damme (1990), "Signalling in a dynamic labour market." The Review of Economic Studies, 57, 1-23. [1106, 1127]

Pancs, Romans (2014), "Designing order book transparency in electronic communication networks." Journal of the European Economic Association, 12, 702-723. [1108]

Swinkels, Jeroen M. (1999), "Education signalling with preemptive offers.” The Review of Economic Studies, 66, 509-528. [1106, 1127]

Co-editor Dilip Mookherjee handled this manuscript.

Submitted 2015-7-7. Final version accepted 2015-10-25. Available online 2015-10-28. 